\title{
Integration of a magnetocaloric heat pump in an energy flexible residential building
}

Johra, Hicham; Filonenko, Konstantin; Heiselberg, Per; Veje, Christian; Dall'Olio, Stefano; Engelbrecht, Kurt; Bahl, Christian

Published in:

Renewable Energy

Link to article, DOI:

10.1016/j.renene.2018.12.102

Publication date:

2019

Document Version

Peer reviewed version

Link back to DTU Orbit

Citation (APA):

Johra, H., Filonenko, K., Heiselberg, P., Veje, C., Dall'Olio, S., Engelbrecht, K., \& Bahl, C. (2019). Integration of a magnetocaloric heat pump in an energy flexible residential building. Renewable Energy, 136, 115-126.

https://doi.org/10.1016/j.renene.2018.12.102

\section{General rights}

Copyright and moral rights for the publications made accessible in the public portal are retained by the authors and/or other copyright owners and it is a condition of accessing publications that users recognise and abide by the legal requirements associated with these rights.

- Users may download and print one copy of any publication from the public portal for the purpose of private study or research.

- You may not further distribute the material or use it for any profit-making activity or commercial gain

- You may freely distribute the URL identifying the publication in the public portal 


\title{
Integration of a magnetocaloric heat pump in an energy flexible residential building
}

\author{
Hicham Johra ${ }^{\text {a, }}{ }^{*}$, Konstantin Filonenko ${ }^{b}$, Per Heiselberg ${ }^{a}$, Christian Veje ${ }^{b}$,
} Stefano Dall'Olio ${ }^{c}$, Kurt Engelbrecht ${ }^{c}$, Christian Bahl ${ }^{c}$

${ }^{a}$ Aalborg University, Division of Architectural Engineering, Department of Civil Engineering, Thomas Manns Vej 23, DK-9220 Aalborg Øst, Denmark

${ }^{\mathrm{b}}$ University of Southern Denmark, Center for Energy Informatics, Campusvej 55, DK-5230 Odense M, Denmark

${ }^{\mathrm{c}}$ Technical University of Denmark, Department of Energy Conversion and Storage, Frederiksborgvej 399, DK-4000 Roskilde, Denmark

*Corresponding author.Tel.: +459940 7234.E-mail address: hj@civil.aau.dk (H. Johra).

\section{Abstract}

The main goal of the ENOVHEAT project is to develop, build and test a prototype of an innovative heat pump based on active magnetic regenerator technology. This device can be coupled to a ground source heat exchanger and an underfloor heating system to provide for the space heating needs of a low-energy house in Denmark. However, the use of a simple controller leads to modest performances because the heating system is running mostly part-load. This numerical study has tested the possibility of using heat storage in the indoor environment and building thermal mass as an effective strategy to improve the operation of the magnetocaloric heat pump. Indoor temperature set point modulation can take advantage of the building energy flexibility potential to maximize the full-load operation time of the heating system and therefore improve its seasonal COP. Results show that this control strategy can significantly increase the seasonal COP, ranging from 2.90 to 3.51 depending on the building thermal mass. Although the indoor temperature stability is reduced, it allows the magnetocaloric heat pump to reach energy use efficiencies which are similar to the ones of conventional vapour-compression heat pumps.

Keywords: Magnetocaloric heat pump; magnetic heating; active magnetic regenerator; innovative heating system; building energy flexibility; demand-side management 


\section{Introduction}

In the recent years, global agreements have been reached concerning the necessity of decreasing our dependency on fossil fuels [1]. To meet these goals, a large deployment of renewable energy sources (RES) and a sharp improvement of our societies' energy efficiency are needed [2]. In many countries, the building sector is the largest energy end-user. In Europe, for instance, it accounts for around $40 \%$ of the total energy demand and indoor space heating represents $75 \%$ of the building energy needs [3]. Consequently, the development of sustainable low-energy buildings with efficient heating systems is essential.

\subsection{Role of heat pump systems}

Heat pumps are mechanical devices which transfer thermal energy from a cold reservoir with low exergy (heat source) to a warmer environment with higher exergy (heat sink) by means of heating/cooling thermodynamic cycles. Conventional heat pumps are typically electrically driven and operate with a vapour-compression thermodynamic cycle. This mature technology for heating is cost effective, presents low $\mathrm{CO}_{2}$ emission, and achieves high coefficient of performance (COP) ranging from 3 to 5 [4][5].

Heat pump systems are therefore an excellent solution to supply heating energy for indoor space conditioning and domestic hot water in countries where the heating demand is dominating. Indeed, in the case of Denmark, a study showed that district heating (in urban areas) and individual heat pumps (outside urban areas) are the best heating supply solutions with regards to costs, energy consumption and $\mathrm{CO} 2$ emissions [6]. Similarly, it was found that heat pumps are an environmentally and economically optimum solution for heating supply in the future RES-dominated energy systems of Germany [7] and the U.K. [8].

Consequently, heat pumps are key components of the energy development strategies in many countries. It therefore leads to a rapid increase of the market demand. For example, in the European Union, between 2005 and 2014, an average of 720000 units were installed each year. In 2014, the total European heat pump stock was of 7.5 million units. With the continuous tightening of the building energy efficiency regulations and the enthusiasm for the renovation sector, the heat pump market presents a significant growth perspective. It is estimated that, if all the European countries had the same market penetration as Sweden, there would be 36.9 million heat pump units running in Europe in 2020 and 85.9 million in 2030 [9].

\subsection{Magnetocaloric technology}

Responding to the large public interest for heat pump technologies, industries and research teams continuously endeavour to develop new cost-effective technical solutions to be production oriented. Numerous technology options could be viable alternatives to the conventional vapour-compression devices for heating, ventilation and air conditioning (HVAC) applications in the built environment. This thriving topic of research and development covers diverse complex physical phenomena leading to technologies with various degrees of potential, maturity and limitation for heating and cooling purpose [10]. Among them, the application of caloric effects in solid refrigerant materials coupled with a sustainable heat transfer fluid is gaining large attention. These caloric effects are caused by a phase transition in the material resulting in a large adiabatic temperature change. Depending on its nature, the caloric effect inside certain materials is induced through a change of a specific parameter of its surrounding environment. The main caloric effects are as follows:

- Electrocaloric effect: adiabatic temperature change by variation of electrical field [11][12]

- Barocaloric effect: adiabatic temperature change by variation of hydrostatic pressure [13]

- Elastocaloric effect: adiabatic temperature change by variation of uniaxial mechanical stress [14][15]

- Magnetocaloric effect: adiabatic temperature change by variation of magnetic field [16][17]

In addition, the concept of the active regenerative cycle can be applied to these caloric effects. It allows the device to transfer heat up to the temperature scale in a useful temperature span for HVAC applications, which is much larger than the sole adiabatic temperature change due to the caloric effect [16].

Among these aforementioned potential alternatives to conventional vapour-compression devices, the magnetocaloricbased technology is currently the most studied and developed of all [18]. The magnetocaloric effect (MCE) is a reversible temperature change occurring in a magnetocaloric material (MCM) when subjected to an adiabatic magnetization or demagnetization. When a magnetic field is applied to the MCM, its magnetic entropy decreases and its temperature increases. Reciprocally, when the MCM is demagnetized, its magnetic entropy increases and its temperature decreases. This phenomenon can be ingeniously employed to create a cooling / heating thermodynamic cycle for heat transfer 
purpose. The reversible nature of the MCE in the solid refrigerant material enables the possibility of developing an efficient heat pump technology with COPs higher than conventional vapour-compression devices. Several types of materials and compounds present a MCE. Their magnetocaloric response is maximum at the ferro-to-paramagnetic phase transition occurring at their respective so-called "Curie temperature". Gadolinium is commonly designated as reference material for the MCE at room-temperature [16][17].

A century ago, the MCE was observed for the first time by Weiss and Piccard [19]. The former was later explained in 1926 by Weiss, Forrer [20] and Debye [21]. It was then suggested by Debye [21] and Giauque [22] that MCE could be used for extremely low temperature cooling purposes. By the mid-1930s, magnetocaloric cooling devices were commonly used in laboratories to achieve absolute temperatures below $1 \mathrm{~K}$ [23]. In 1976, Brown experimentally demonstrated that a Gadolinium-based magnetocaloric device could be used for near room-temperature heating / cooling applications [24]. A major breakthrough was achieved by Barclay and Steyert when they developed and patented the active magnetic regenerator (AMR) cycle in 1982 [25]. With this configuration, the magnetocaloric solid refrigerant is used as a thermal regenerator. This allows a simplification of the device and a significant increase of the temperature span above the adiabatic temperature change induced by the MCE alone [16]. The AMR design is considered to be the most thermodynamically efficient for magnetic heating / cooling devices [26]. Consequently, the AMR configuration has been the foundation principle of almost all the magnetic cooling / heating systems ever since [16].

In the following decades, the magnetic heating / cooling technology gained popularity. Multiple laboratories and research groups built and tested their own AMR prototypes. Zimm et al. reported in 1998 that their near room-temperature magnetic refrigerator using superconducting magnets could operate with COPs above 6 [27]. A large MCE can be generated with the use of superconducting magnets, but these are costly and can be impractical for HVAC applications in buildings. Therefore, most of the recent AMR prototypes are using permanent magnets. In 2012, the rotary AMR device of Engelbrecht et al. was operating with a no-load temperature span of $25.4 \mathrm{~K}$ and a maximum cooling capacity of 1010 W [28]. In 2013, Okamura and Hirano tested a magnetic refrigerator operating at a COP of 2.5 with a temperature span of $5 \mathrm{~K}$ [29]. In 2014, Jacobs et al. presented a prototype generating $2502 \mathrm{~W}$ of cooling power with a $12 \mathrm{~K}$ temperature span and a COP above 2. In addition, the machine reached up to $3042 \mathrm{~W}$ of cooling power at a zero-temperature span [30]. In 2015, an Italian research group investigated experimentally a magnetic refrigerator coupled directly to a vertical borehole ground source heat exchanger (GSHE). The device could produce cold water below $15^{\circ} \mathrm{C}$ for a cooling capacity of $190 \mathrm{~W}$ at a COP of 2.2 [31]. In another publication, the same research group indicated that this AMR device could operate at a maximum no-load temperature span of $11.9 \mathrm{~K}$, and a maximum COP of 2.5 for a thermal load of $200 \mathrm{~W}$ [33]. In 2016, a Brazilian team reported that their magnetocaloric device had a maximum zero-span cooling power of $150 \mathrm{~W}$ and a maximum no-load temperature span of $12 \mathrm{~K}$. With a thermal load of $80.4 \mathrm{~W}$, the machine generated a $7.1 \mathrm{~K}$ temperature span with a COP of 0.54 [33]. The same year, a research laboratory at the Technical University of Denmark presented an AMR device capable of reaching $81.5 \mathrm{~W}$ of cooling power with a COP of 3.6 and a $15.5 \mathrm{~K}$ temperature span [34].

A comprehensive explanation of the magnetocaloric effect, materials and systems can be found in the publications of Smith et al. [16] and Kitanovski et al. [17]. The AMR system principle is described in detail in the articles of Engelbrecht et al. [28] and Lei et al. [35]. 
3 In line with the global endeavour to improve heating and cooling systems, the main goal of the ENOVHEAT 4 project [36] is to develop, build and test the prototype of an innovative heat pump for building applications based on 5 AMR technology (see Fig. 1). In addition to great potential for high COPs, AMR-based devices have the benefit of a low 6 noise level operation, an absence of greenhouse or toxic gases, and the possibility for recycling the magnets and the 7 magnetocaloric materials [16]. 


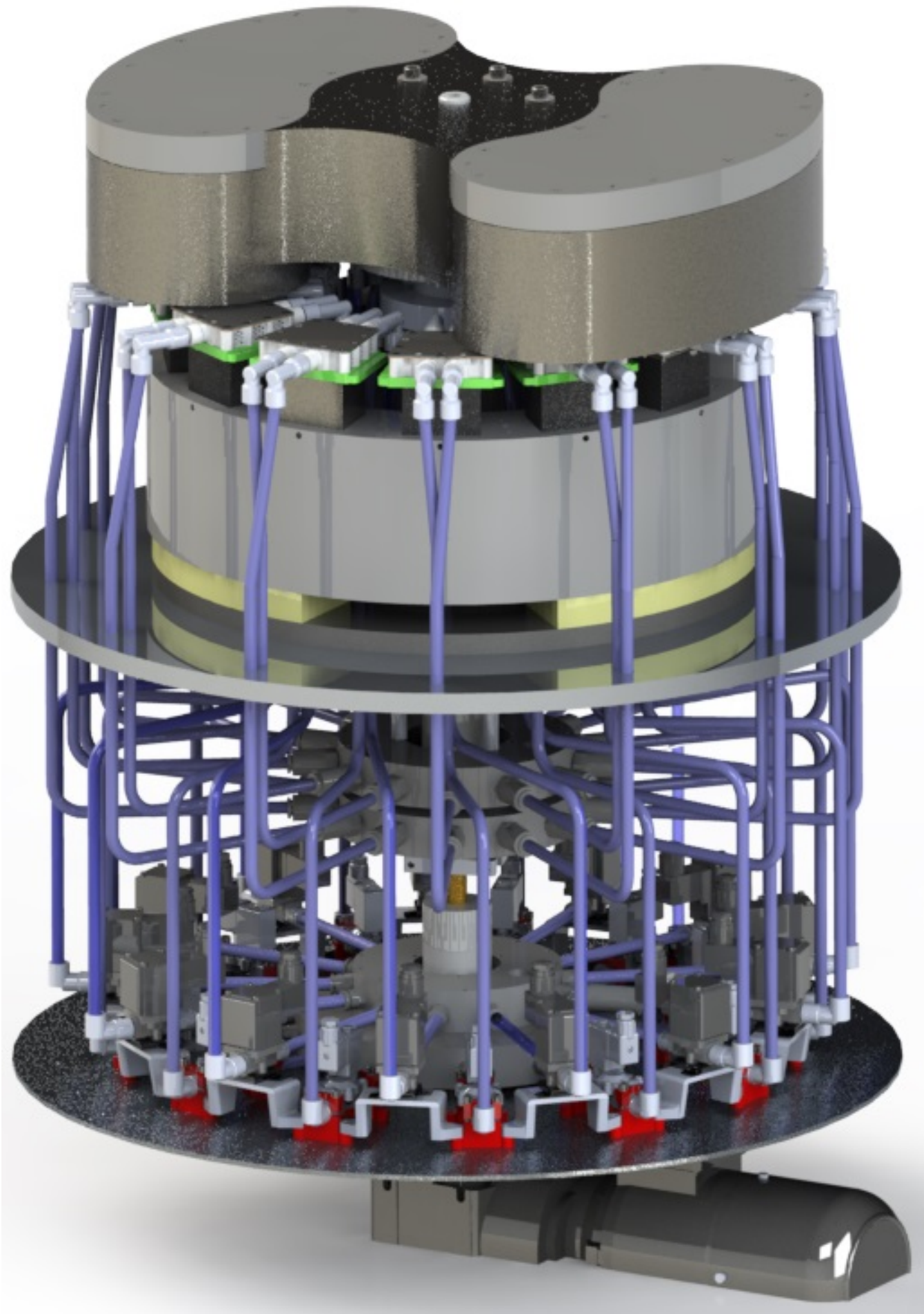

Fig. 1. CAD model of the magnetocaloric heat pump prototype of the ENOVHEAT project: "MagQueen". 
A previous study from the ENOVHEAT project [37] numerically demonstrated for the first time that such a magnetocaloric heat pump (MCHP) can be implemented in a low-energy single family house and provide for its space heating needs under cold weather climate conditions (Denmark). In this project, the MCHP is used solely for indoor space heating because domestic hot water production requires a higher temperature span. Nevertheless, this is an encouraging starting point for the development of this promising technology for building applications.

As shown in the GeoThermag project [31], a MCHP can be directly coupled with a GSHE. Similarly, in the ENOVHEAT project, a MCHP is integrated in a single hydronic loop including a vertical borehole GSHE and a radiant under-floor heating (UFH) without an intermediate heat exchanger or hot water storage tank. As shown in Fig. 2, this specific MCHP system configuration can run with appreciable COPs. It should be noted that these results are taking into account all losses and inefficiencies from the entire heating system and GSHE. Therefore, the results of the Fig. 2 can look different from the ones of publications reporting COPs of the sole AMR operation. The MCHP of the current study can produce $2600 \mathrm{~W}$ of useful heating power with an average seasonal COP of 3.93 when operating at fluid flow rate of $2200 \mathrm{~L} / \mathrm{h}$ and with a rotation frequency of $1 \mathrm{~Hz}$ [37].

One can also observe in Fig. 2 that the system's COP drops dramatically above an optimum fluid flow rate. This is a typical behaviour for AMR-based systems [38][39][40]. In the case of passive thermal regenerators, small utilization factors associated to low fluid flow rates are favourable to obtain high regenerator effectiveness. However, for AMR, utilization factor and fluid flow rate must be adjusted according to the device's characteristics and operation conditions in order to achieve optimal performance. At low fluid flow rates, despite the temperature span being significant, the small amount of heat transfer fluid passing through the active regenerator cannot produce a significant heating power. Then the heating power output and COP of the MCHP increase with the fluid flow rate until reaching an optimum point. Above this optimum point, further increase of the fluid flow rate disturbs the temperature profile of the AMR. This causes a drop of the temperature span and, consequently, a collapse of the MCHP heating power output. In addition, a higher fluid flow rate induces higher pressure losses in the hydraulic system, which also lessens the system's COP. In order to increase the maximum achievable heating power at a higher fluid flow rate, it is necessary to increase the operation frequency of the MCHP. At a higher operating frequency, more AMR cycles are performed over a given period of time. Therefore, the rate of magnetic work performed on the MCM is increased together with the maximum power output. However, operating efficiently a MCHP at higher frequencies leads to new technical challenges [40].

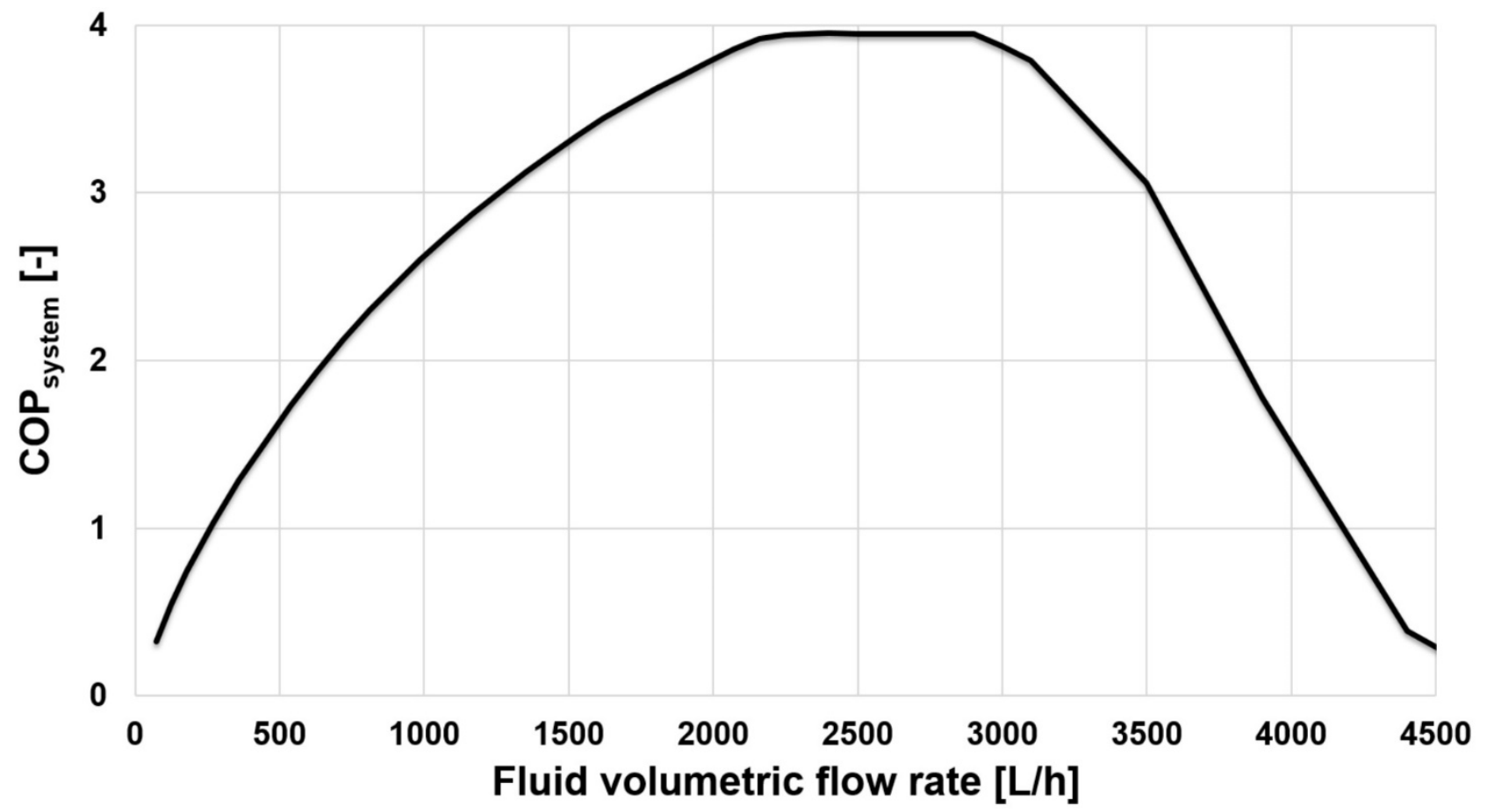

Fig. 2. COP of the entire heating system (comprising the MCHP, the vertical borehole GSHE and the UFH hydronic loops) as a function of the heat pump fluid volumetric flow rate [37]. 
In addition, if the MCHP is implemented in a multi-zone building with a simple fluid flow rate controller and independent thermostats in each thermal zone, the heating system operates on partial-load most of the time. As illustrated in Fig. 3, this leads to modest performances (average seasonal COP of 1.84) compared to the optimum operation point of this MCHP [37]. The development of control strategies adjusting fluid flow and operation frequency of the MCHP for efficient partial-load operation is thus very important [41].
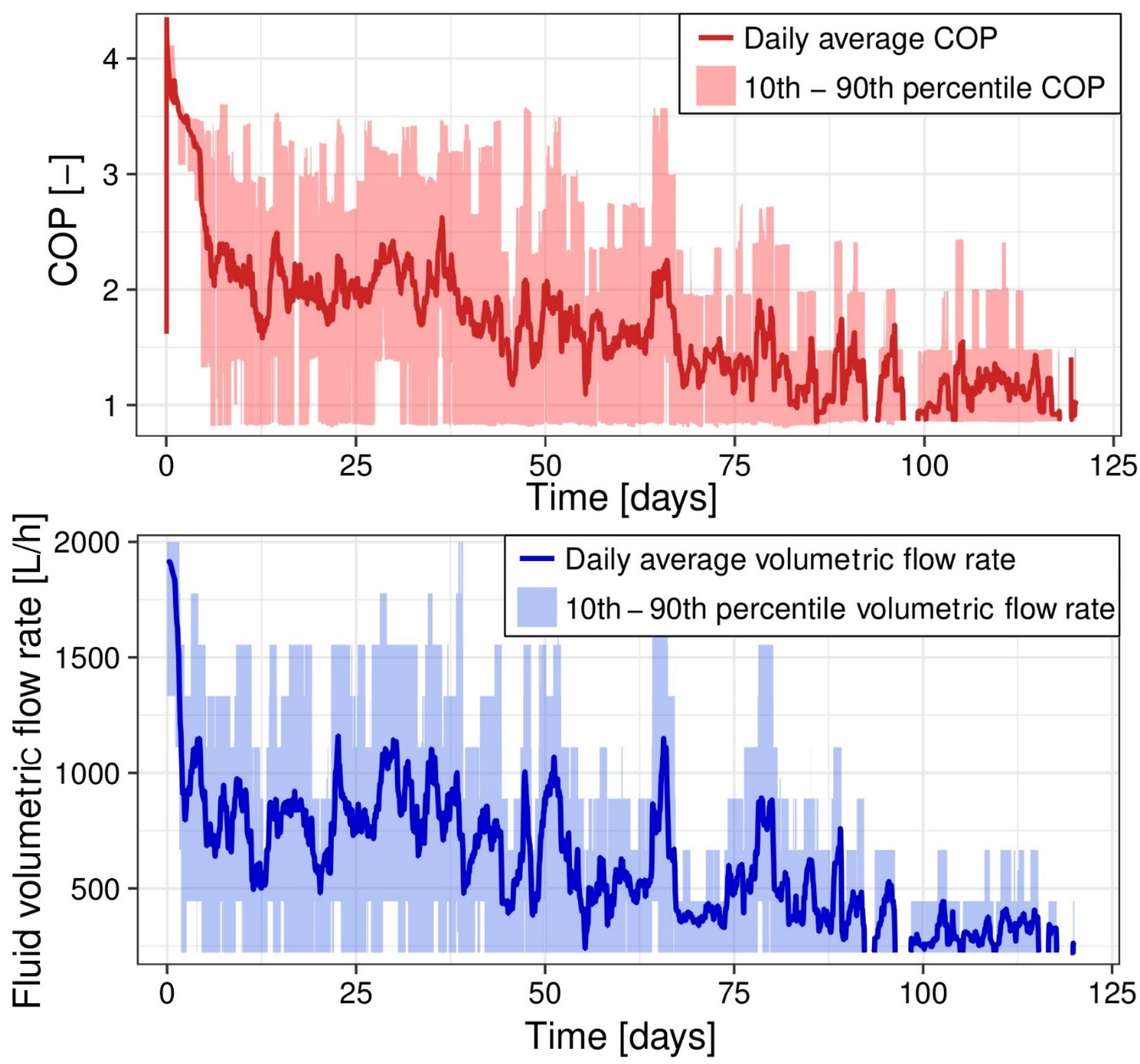

Fig. 3. COP and fluid volumetric flow rate of the MCHP heating system during a four-month winter test period [37]. 
The major drawback of RES is the intermittence of power generation. The increasing share of RES in electricity grids can thus induce major mismatch between instantaneous energy use and production, leading to grid instability. A paradigm shift is occurring in the field of energy system management and the concept of Smart Grids with massive RES penetration is emerging. The future Smart Grid systems are energy grid networks which can intelligently integrate the actions of all users connected and sending information to them, in order to efficiently provide sustainable and reliable energy supplies. These grid users are the energy producers, energy suppliers, energy end-users (which can also be energy producers: "prosumers"), and energy storage systems [42]. Consequently, the continuous increase of intermittent RES in the global energy mix induces a fast growing demand for energy storage and energy end-user flexibility [43]. For that matter, the building sector should not be considered as a simple passive end-user but, on the contrary, as a major active player which can help regulating the electricity production and consumption. Buildings' energy demand can be modulated by means of thermal storage, HVAC usage adjustment, electric vehicles charging scheduling, plug-loads shifting, etc. These demand side management measures are commonly denominated as "Building Energy Flexibility" strategies [44].

As mentioned before, the heating need for indoor space conditioning is a major target for building efficiency improvement. It is also the case for building energy flexibility strategies. The large potential for thermal energy storage (TES) in buildings can be cleverly employed to shift heating use in time and thus reshape the overall power profile. To that end, it was found that passive TES in the indoor environment and building structural thermal mass is more cost effective than enlarging storage water tanks [45]. In conventional vapour-compression heat pump (VCHP) systems, most of the flexibility is provided by an accumulation hot water tank. However, there is no water tank in the current MCHP system implementation [37]. Indoor temperature set point modulation for passive TES in the indoor space could therefore be a solution to enable building energy flexibility [46] and improve the MCHP operation by increasing its running time at highest COP.

\subsection{Aim of the current study}

The current numerical study extends the previous research conducted on the integration of this innovative heat pump in dwellings [37] by implementing a new control strategy taking advantage of the building energy flexibility potential. Firstly, the MCHP system and the building study cases are described. The heat storage control strategy for improved heating system performances is then presented. Finally, the benefits of this control strategy concerning the heat pump operation are discussed and followed by a conclusion and suggestions for further work on that research topic. 


\subsection{Characteristics of the magnetocaloric heat pump}

In this study, the MCHP is a rotary AMR-based device (see Fig. 4). The refrigerant is a solid MCM (Gadolinium) arranged as packed bed sphere $(450 \mu \mathrm{m}$ diameter) in trapezoidal shaped-cassettes regenerators (see Fig. 5). The tapered regenerators have a length of $59 \mathrm{~mm}$, a height of $17 \mathrm{~mm}$, an average width of $61.43 \mathrm{~mm}$, and a gradient angle of -10 degrees [39]. Gadolinium is a well-known rare-earth element experiencing a large adiabatic temperature change when magnetized in the vicinity of its Curie temperature (around $293 \mathrm{~K}$ ). As mentioned before, Gadolinium is the reference material for MCE at room-temperature [16]. The MCHP contains $2.8 \mathrm{~kg}$ of Gadolinium in total. 13 regenerators are mounted on an iron ring constituting the vertical stator. The vertical rotor is a two-pole magnet assembly with an iron yoke (see Fig. 6). Each pole is composed of 28 permanent magnet elements. A vertical shaft connects the rotor to an electric motor. The rotation of the magnets creates a varying magnetic field and induces alternatively magnetization and demagnetization of the MCM in the regenerators. The magnetic field has a maximum value of $1.46 \mathrm{~T}$ in the high air-gap. The rotation frequency of the device is fixed at $1 \mathrm{~Hz}$.

The 13 AMRs are connected to two manifold collectors and two manifold distributors. One collector and one distributor are on the cold side of the AMRs and similarly on the hot side. For each of the regenerator beds there are two solenoid valves synchronized with the position of the magnet via an absolute encoder. These valves allow for bidirectional circulation of the coolant fluid (20\%vol ethylene glycol and $80 \%$ vol water) through the individual MCM porous media beds while the overall flow to the device is delivered by a continuously operating pump. The global effect is to transfer thermal energy from the cold side (connected to the heat source) to the warm side (connected to the heat sink) of the regenerators. A single centrifugal pump circulates the heat transfer fluid from the heat source to the two manifold distributors and from the two manifold collectors to the heat sink. The fluid flow rate in the MCHP is limited to $2200 \mathrm{~L} / \mathrm{h}$.

The main parameters of the MCHP of this numerical study are summarized in Table 1. A detailed description of the ENOVHEAT MCHP device and its operation principle can be found in the paper published by Johra et al. [37]. 


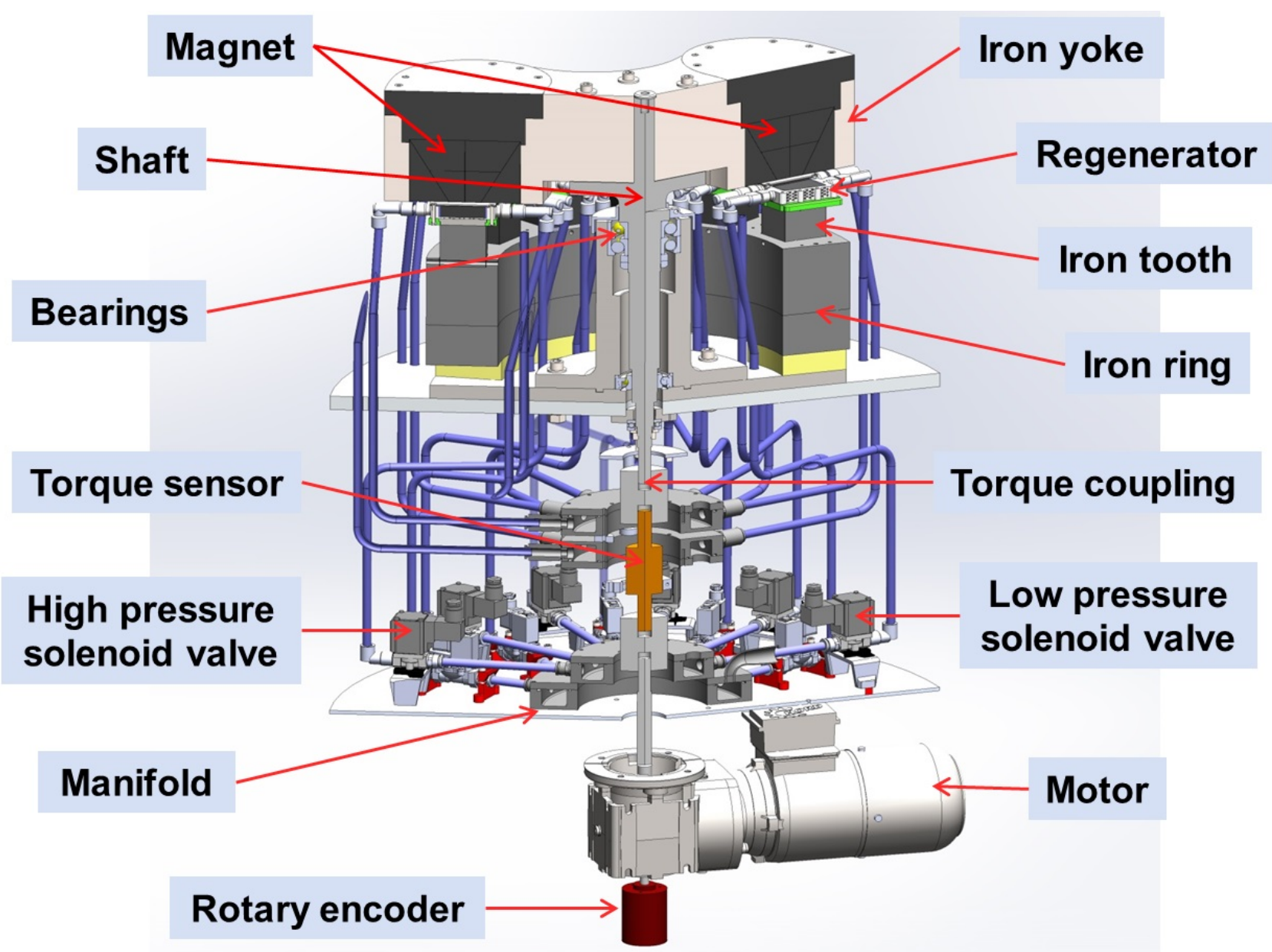

Fig. 4. Detailed description of the magnetocaloric heat pump prototype of the ENOVHEAT project: "MagQueen".
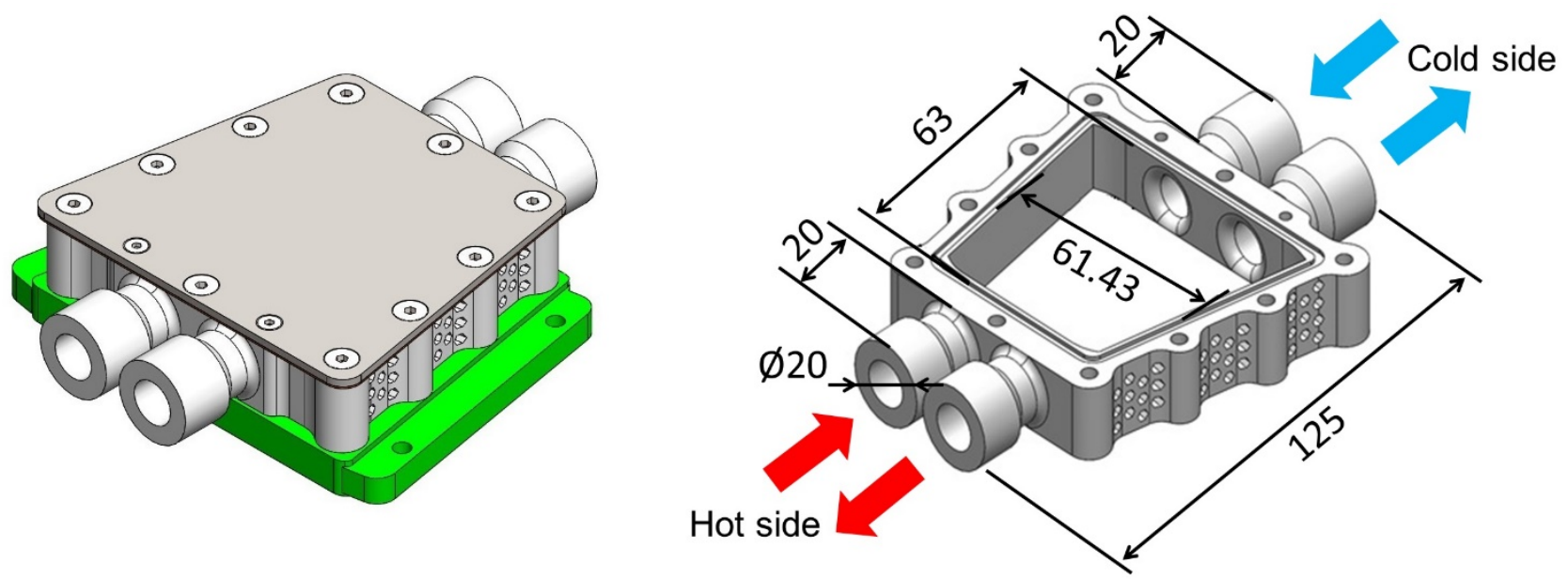

Fig. 5. CAD model of the regenerator containing the MCM bed (dimensions are indicated in millimeters). 

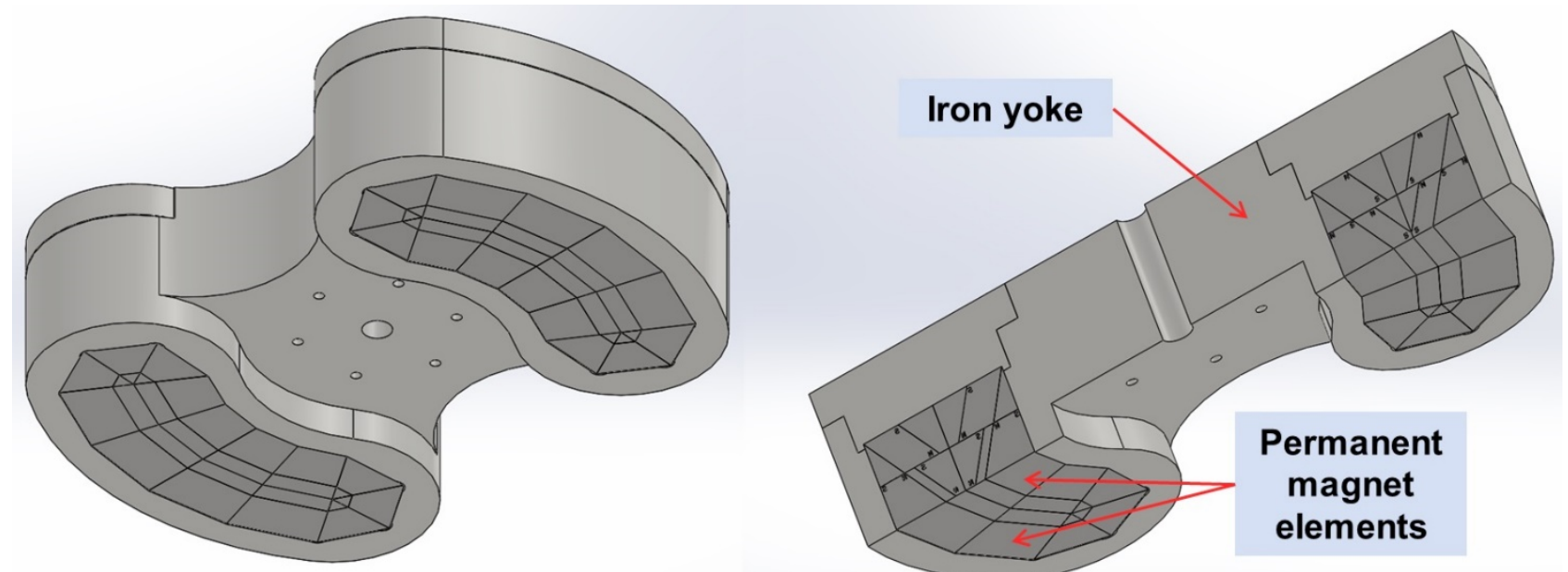

Fig. 6. CAD model of the two-pole magnet assembly.

2

\begin{tabular}{|c|c|}
\hline Parameter & Value \\
\hline Magnetocaloric heat pump configuration (-) & Static AMR - Rotating magnets \\
\hline Magnetocaloric material (-) & Gadolinium (Gd) \\
\hline MCM Curie temperature (K) & 293 \\
\hline Total mass of MCM $(\mathbf{k g})$ & 2.8 \\
\hline Number of regenerators $(-)$ & 13 \\
\hline Regenerator length $(\mathrm{mm})$ & 59 \\
\hline Regenerator height $(\mathbf{m m})$ & 17 \\
\hline Regenerator average width $(\mathrm{mm})$ & 61.43 \\
\hline Regenerator gradient angle $\left(^{\circ}\right)$ & -10 \\
\hline Regenerator bed geometry (-) & Packed sphere bed \\
\hline Sphere diameter $(\mu \mathrm{m})$ & 450 \\
\hline Maximum applied magnetic field $(\mathrm{T})$ & 1.46 \\
\hline Rotation frequency $(\mathrm{Hz})$ & 1 \\
\hline Fluid volumetric flow rate (L/h) & $0-2200$ \\
\hline
\end{tabular}




\title{
2.2. Numerical modelling of the magnetocaloric heat pump
}

Originally, the MCHP has been modelled by Engelbrecht [38] and then further developed by Lei et al. [35]. If reasonable assumptions on regenerator's geometry, external heat losses and demagnetization losses are made, the timedependent fluid temperature distribution in the AMR can be calculated with two coupled partial differential equations:

$$
\begin{gathered}
\frac{\partial}{\partial x}\left(k_{\text {disp }} A_{c} \frac{\partial T_{f}}{\partial x}\right)-\dot{m}_{f} c_{f} \frac{\partial T_{f}}{\partial x}-\frac{N u k_{f}}{d_{h}} a_{s} A_{c}\left(T_{f}-T_{s}\right)+\left|\frac{\partial P}{\partial x} \frac{\dot{m}_{f}}{\rho_{f}}\right|=A_{c} \varepsilon \rho_{f} c_{f} \frac{\partial T_{f}}{\partial t} \\
\frac{\partial}{\partial x}\left(k_{\text {stat }} A_{c} \frac{\partial T_{s}}{\partial x}\right)+\frac{N u k_{f}}{d_{h}} a_{s} A_{c}\left(T_{f}-T_{s}\right)=A_{c}(1-\varepsilon) \rho_{s} \times\left[c_{H} \frac{\partial T_{s}}{\partial t}+T_{s}\left(\frac{\partial s_{s}}{\partial H}\right)_{T_{s}} \frac{\partial H}{\partial t}\right]
\end{gathered}
$$

\begin{abstract}
Where $k, T, \rho, c$ and $s$ are the thermal conductivity, temperature, density, specific heat, and specific entropy; $A_{c}, d_{h}$
\end{abstract} , $a_{s}, \varepsilon, x, t, \dot{m}_{f}$, and $H$ are the cross sectional area, hydraulic diameter, specific surface area, porosity of the regenerator bed, axial position, time, fluid mass flow rate and internal magnetic field; $\partial P / \partial x$ and $N u$ are the pressure drop and the Nusselt number. The subscripts $f$ and $s$ represent fluid and solid refrigerant, respectively. $k_{\text {disp }}$ is the thermal conductivity of the fluid due to axial dispersion, $k_{\text {stat }}$ is the static thermal conductivity of regenerator and fluid, and $c_{H}$ is the specific heat capacity of the MCM at constant magnetic field. An implicit finite volume method scheme is used to solve these equations within the MATLAB software environment. This numerical model has been successfully validated with experimental data of a real AMR device [47]. For the purpose of the numerical simulation of an entire building, the detailed MCHP model is approximated by a series of around 1600 quasi-steady states implemented in 5-dimensional lookup tables. These lookup tables are function blocks of the MATLAB-Simulink software environment which can generate the MCHP output values with minimum computation needs. The four input variables of the simplified MCHP model are rotation frequency, volumetric fluid flow rate, cold side temperature inlet and hot side temperature inlet. The outputs of the simplified MCHP model are cold side and hot side outlet fluid temperatures, magnetic work and fluid pressure losses through the regenerators. The other components of the MCHP (valves, motor, circulation pump) are modelled with data from measurements on the ENOVHEAT prototype and from manufacturers documentation. The average electrical power of the set of solenoid valves is $63 \mathrm{~W}$. The motor work is calculated from the magnetic work of the AMR device and assuming a motor efficiency of 0.65 . The pump work is calculated with a polynomial function fitting the operation data provided by the manufacturer [37].

More details about the numerical modelling of the ENOVHEAT MCHP can be found in the paper published by Johra et al. [37].

\section{Building systems}

\subsection{Building study case}

The building study case of the ENOVHEAT project for testing the integration of the MCHP is a single family house in Denmark. This single-story dwelling has a heated floor surface area of $126 \mathrm{~m}^{2}$ and a geometry which is typical for modern Danish houses (see Fig. 7). It is a low-energy building with a yearly heating need of $16 \mathrm{kWh} / \mathrm{m}^{2}$ [48].

In the case of TES in the indoor environment, the building envelope performance and the structural thermal inertia are the main parameters determining the heat storage capacity and efficiency of a building and, consequently, its energy flexibility potential [49]. However, the current configuration of the MCHP is restricted to low-energy buildings. Therefore, only the structural thermal inertia of the house is varied to assess the influence of the energy flexibility on the MCHP performance. three different classes of structural thermal inertia are defined for this house:

- Light-weight structure building: daily effective thermal inertia of $30 \mathrm{Wh} / \mathrm{K} \cdot \mathrm{m}^{2}$.

- Medium-weight structure building: daily effective thermal inertia of $60 \mathrm{Wh} / \mathrm{K} . \mathrm{m}^{2}$.

-Heavy-weight structure building: daily effective thermal inertia of $100 \mathrm{Wh} / \mathrm{K} \cdot \mathrm{m}^{2}$.

The outdoor boundary conditions for air and ground temperature, solar gains, atmospheric pressure, cloud cover, relative humidity, wind speed and long wave radiation are extracted from the weather file of the Danish Reference Year (DRY 2013) [50]. It is assumed that four persons are living in the dwelling with occupancy following a representative weekly schedule for Denmark. The equipment and people load time distribution are set accordingly [51]. 
1 More details about the ENOVHEAT building study cases can be found in the paper published by Johra et al. [37] and 2 in a DCE technical report [52].

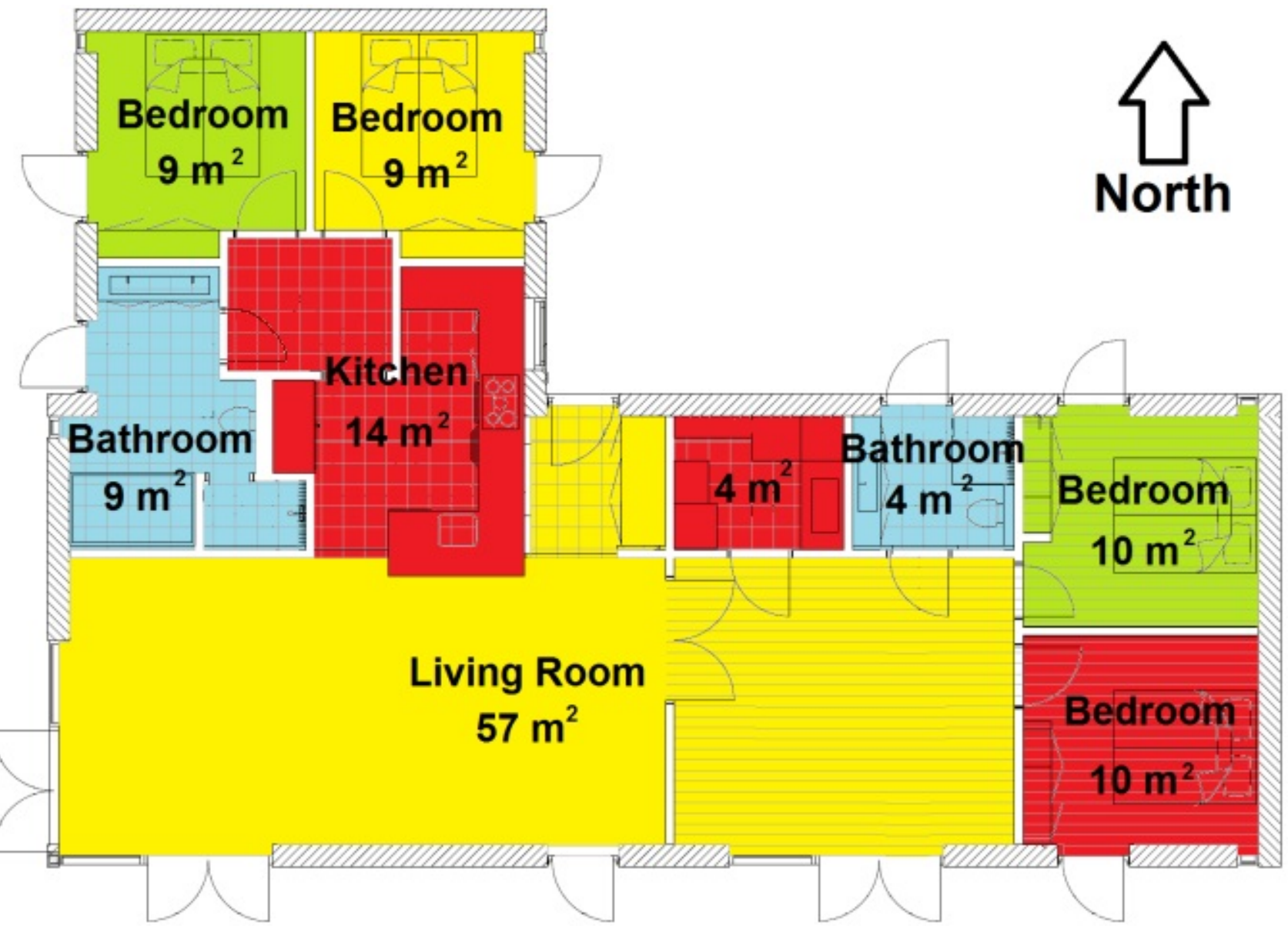

Fig. 7. Plan view of the house study case. 


\subsection{Heating systems}

To maximize the performance of the simulated heat pump system, a vertical borehole GSHE is used as high temperature heat source, and a hydronic radiant under-floor heating system is used as low temperature heat sink. The under-floor heating system consists of PE-Xa pipe (16 mm outer diameter) loops embedded in a $100 \mathrm{~mm}$ thick concrete screed in the case of the medium and heavy thermal inertia houses. For the light thermal inertia house cases, UFH pipes are embedded in the wooden floor. It has been sized according to international standards and manufacturer's guidelines [53][54][55]. The ground source loop is a vertical borehole heat exchanger consisting of a single collector double U-tube PEX pipe (44 mm diameter). The borehole is $100 \mathrm{~m}$ deep and has a diameter of $160 \mathrm{~mm}$. The total pipe length of the ground loop is thus $200 \mathrm{~m}$. The design and sizing of the GSHE has been made according to international standards and manufacturer's guidelines [56][57][58], and assuming that the soil is a humid clay sand with a thermal conductivity of 1.5 $\mathrm{W} / \mathrm{m} . \mathrm{K}$, a density of $1900 \mathrm{~kg} / \mathrm{m}^{3}$ and a specific heat capacity of $1400 \mathrm{~J} / \mathrm{kg} . \mathrm{K}$. The grouting material of the borehole is assumed to have a thermal conductivity of $1.4 \mathrm{~W} / \mathrm{m} . \mathrm{K}$, a density of $1500 \mathrm{~kg} / \mathrm{m}^{3}$ and a specific heat capacity of 1670 $\mathrm{J} / \mathrm{kg} . \mathrm{K}$.

The MCHP operates at temperatures and fluid flow rates which are compatible with direct use in the heat emitter. Therefore, the GSHE and the UFH are coupled to the MCHP inside a single hydronic loop without any intermediate heat exchanger or hot water storage tank (see Fig. 8). With that configuration, the same heat transfer fluid ( $20 \%$ vol ethylene glycol and $80 \% \mathrm{vol}$ water) is circulated through the heat source, the heat sink and the MCHP. The fluid flow is generated by the internal circulation pump of the MCHP. The volumetric fluid flow rate is limited to a maximum of $2200 \mathrm{~L} / \mathrm{h}$ in the MCHP and in the GSHE, and around $240 \mathrm{~L} / \mathrm{h}$ in each UFH sub-circuit. This fluid flow rate limitation prevents excessive pressure losses in the hydraulic system (see Fig. 9), pump work and wear and tear of pipes and fittings, but also ensure that no vibration or noise occurs in the piping inside the building.

The original controller for the MCHP heating system is a basic flow rate regulation. The speed of the circulation pump is adjusted to keep a nominal fluid volumetric flow rate of around the maximum limit in each UFH sub-circuits. There are nine UFH hydronic loops arranged in the eight thermal zones of the house. Each thermal zone has a thermostat adjusting the indoor temperature to a given set point by opening/closing a motorized valve with an ON/OFF controller.

The performance of the MCHP is compared with a conventional water-to-water VCHP implemented in the same building case with the same UFH system and vertical borehole GSHE. The VCHP has characteristics similar to the model TWM036 of ClimateMaster ${ }^{\circledR}$ [59]. With a nominal fluid flow rate of $2052 \mathrm{~L} / \mathrm{h}$ in both heat source and heat sink, this ground source VCHP has a heating power output of $8.28 \mathrm{~kW}$ with a COP of 4.51 (heat source at $5{ }^{\circ} \mathrm{C}$ and heat sink at 35 ${ }^{\circ} \mathrm{C}$ ). A $250 \mathrm{~L}$ hot water storage tank, 3 circulation pumps and a mixing valve are added to the heating system (see Fig. 10). The hot water accumulation tank has a temperature set point of $35^{\circ} \mathrm{C}$. The water temperature is measured in the top third of the water tank.

More details about the building heating systems can be found in the paper published by Johra et al. [37] and in a DCE technical report [52]. 


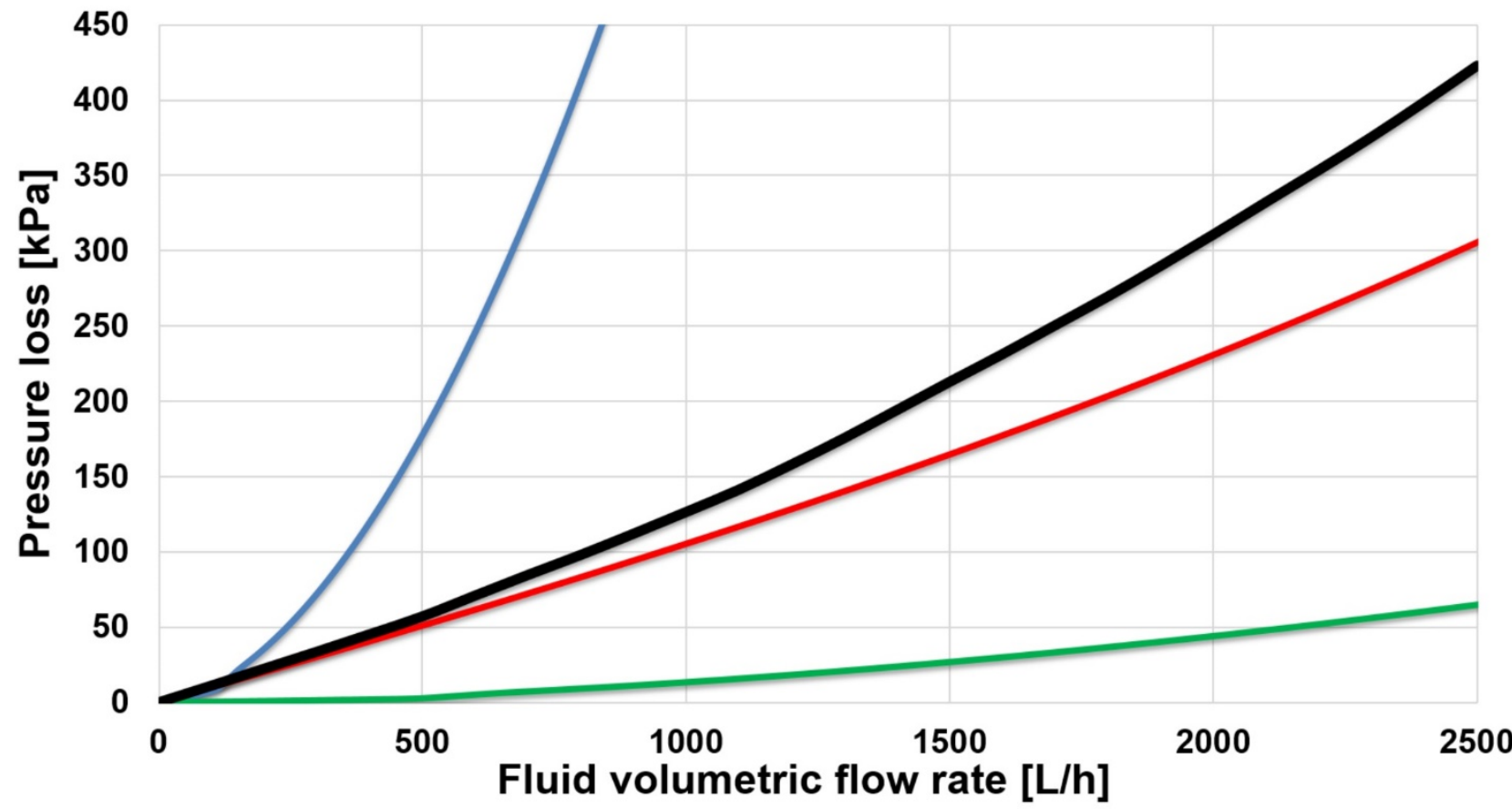

Fig. 8. Integration of a magnetocaloric heat pump in single hydronic loop with ground source heat exchanger and under-floor heating system.

\section{Magnetocaloric heat pump}

Room temperature

Building

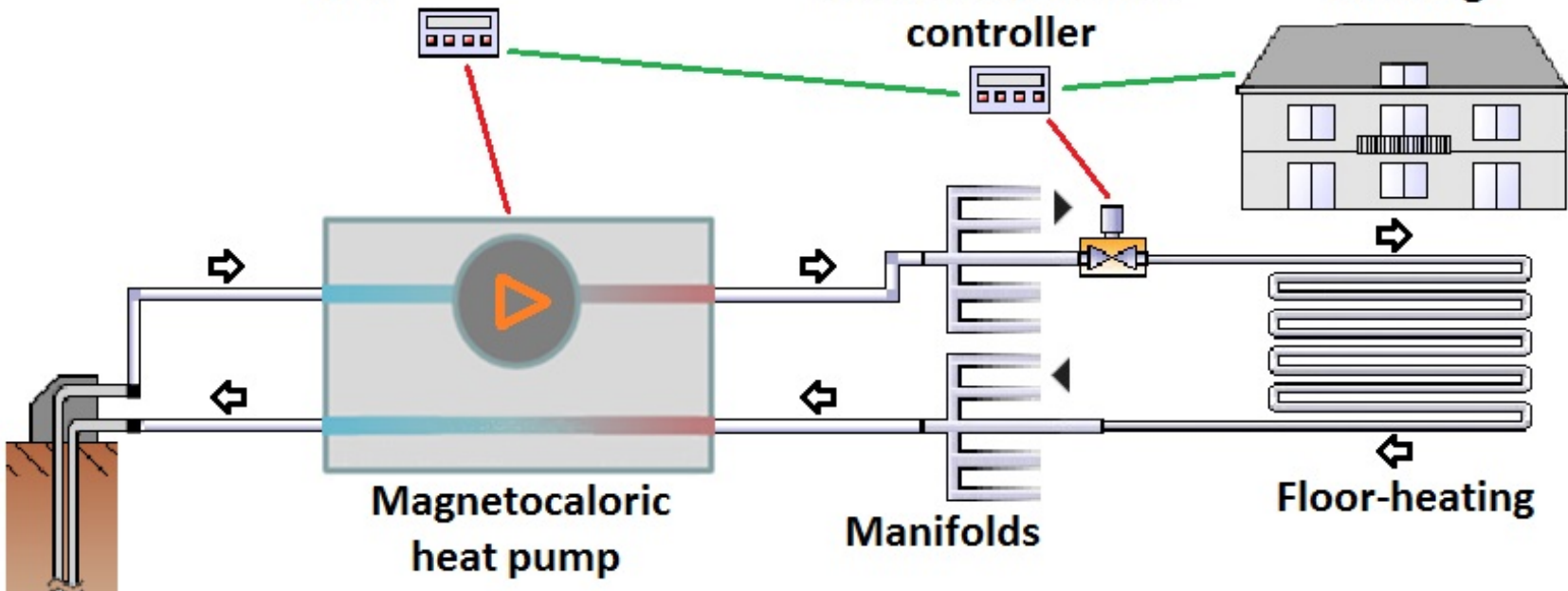

\section{Ground-source}




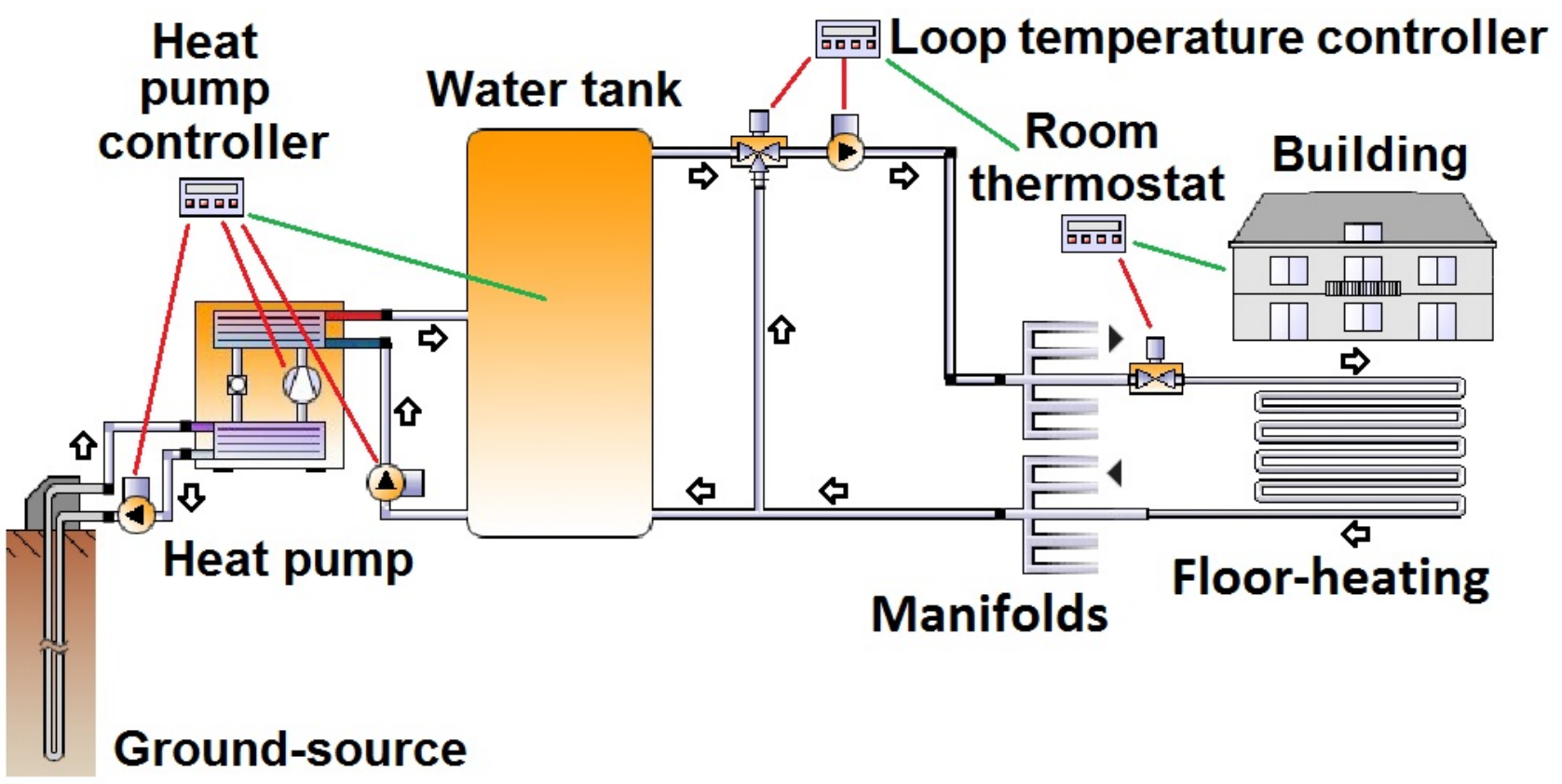

Fig. 10. Integration of a conventional vapour-compression heat pump with ground source heat exchanger, hot water storage tank and under-floor heating system. 
A thermodynamic multi-zone model of the building is created within the MATLAB-Simulink software environment. A one-dimensional explicit finite volume method with a limited number of control volumes (commonly named "Resistance-Capacitance thermal network" or "RC network") is employed to calculate the heat transfer through the construction elements by solving the heat equation:

$$
\rho c \frac{\partial T}{\partial t}=\nabla \cdot(k \nabla T)+q_{V}
$$

Where $\rho, \mathrm{c}, \mathrm{T}, \mathrm{k}$ and $q_{V}$ are the density, specific heat capacity, temperature, thermal conductivity and volumetric heat source, respectively. Surfaces facing the indoor space are connected to the indoor air node within a star network configuration using constant mixed convection/radiation thermal resistance coefficients. Constant thermal resistances are used to model thermal bridges, ventilation, air infiltration and windows heat losses. Solar heat gains and long-wave radiation to the sky are calculated as a function of surface orientation and outdoor weather conditions.

The hydronic under-floor heating is modelled with the $\varepsilon$-NTU method [54][60] as a horizontal heat exchanger embedded in a multilayer slab. The heat transfer from the brine fluid to the surrounding environment of the heat exchanger is calculated with the following equations:

Where $\mathrm{U}, R_{f}, R_{p}$ and $R_{x}$ are the heat exchanger heat transfer coefficient, thermal resistance of the fluid, thermal resistance of the pipe and thermal resistance of interaction between the tubes of the heat exchanger, respectively. NTU, $\mathrm{A}, \dot{m}_{f}$ and $c_{f}$ are the number of transfer units, heat transfer area, fluid mass flow rate and fluid specific heat capacity, respectively. q, $\varepsilon, T_{f}$ and $T_{x}$ are the heat transfer from the fluid to the surrounding of the heat exchanger, effectiveness of the heat exchanger, inlet fluid temperature and heat exchanger temperature, respectively.

The dynamics of the fluid in the pipe network when the flow rate is varying are simulated with a "plug flow" model [61]. The vertical borehole GSHE component is created by coupling two straight pipe heat exchanger sub-models ( $\varepsilon$-NTU method with "plug flow" model) in a triangular thermal network (Resistance-Capacitance network) [62]. The underground soil around the ground source is considered as a one-dimensional finite domain and implemented in a MATLAB state space function. Temperature boundary conditions are defined on the upper ground surface by the weather conditions. At a depth of $100 \mathrm{~m}$, the ground temperature is set constant to $10.1^{\circ} \mathrm{C}$. The thermo-physical properties of the brine and the associated convective heat transfer coefficient and viscosity in the pipes are calculated as a function of the fluid composition, velocity and temperature. Pressure losses in pipes caused by fluid friction are calculated with the Darcy-Weisbach equation:

$$
\Delta p=\frac{\rho \times v^{2}}{2}\left(\frac{f \times L}{D}+K_{i}\right)
$$

Where $\Delta p, \rho, \mathrm{v}, \mathrm{f}, \mathrm{L}, \mathrm{D}$ and $K_{i}$ are the pressure loss, the fluid density, the fluid average velocity, the friction factor, the length of the pipe, the internal diameter of the pipe and the singular friction factor of additional elements, respectively.

Similarly to the MCHP, the conventional VCHP is modelled with a collection of steady states implemented in a 4dimensional lookup table function. The lookup table data is obtained from documentation of the VCHP manufacturer [59]. The $250 \mathrm{~L}$ hot water storage tank has a cylindrical shape (radius of $29 \mathrm{~cm}$; height of $95 \mathrm{~cm}$ ) with $5 \mathrm{~cm}$ of polyurethane 
insulation (heat losses to the ambient are $1.356 \mathrm{~W} / \mathrm{K}$ ). The water tank model is a simplified version of that presented by Angrisani et al. [63].

The building model has been validated with a BESTEST procedure [64]. Sub-components of the building model have been validated against commercial software or experimental data. More details about the numerical models of the building systems can be found in the paper published by Johra et al. [37]. Validation test results and detailed description of the building sub-components can be found in a DCE technical report [52].

\section{Heat storage control strategy}

As mentioned before, the original MCHP controller is simple and causes the heating system to operate with modest performances. This simple control strategy is based on a basic fluid flow regulation. Each of the UFH sub-circuit is equipped with a valve regulated by an ON/OFF controller. The valve is fully open (fluid volume flow rate of around 240 $\mathrm{L} / \mathrm{h}$ ) when the operative temperature of the room is below the indoor temperature set point. The valve is closed when the operative temperature of the room is above the temperature set point. The speed of the circulation pump and the fluid flow in the MCHP are adjusted accordingly [37]. However, each room in the house has very different heating need profiles because of different internal people load, equipment load and solar load. It is thus very rare that all rooms require maximum heating power at the same time. Consequently, the MCHP does not run at the optimum fluid flow rate and highest COP most of the time.

A new control strategy is thus implemented. It takes advantage of the building energy flexibility potential by using indoor temperature set point modulation to allow TES in the indoor environment. The set point temperature is the same in all rooms of the house. The set points are changed all together at the same time in all rooms. When the heating system is activated, it operates at high capacity with optimum COP to store thermal energy in the house's thermal mass leading to a slight increase of the indoor temperature. When the building is fully charged, the heating system is completely turned off and the indoor temperature freely decreases until reaching a critical threshold for re-activation of the heat pump. The maximization of the MCHP operation time at optimum COP increases the overall performance of the heating system.

Fig. 11 illustrates in more details this new heat storage strategy and compares it to the simple controller (building case study with high thermal inertia: $100 \mathrm{Wh} / \mathrm{K} . \mathrm{m}^{2}$ ). One can see in Fig. 11(a) that the house indoor temperature is allowed to vary around the "neutral temperature set point" of $22^{\circ} \mathrm{C}$. When the lowest temperature of the house (coldest room) reaches the "minimum temperature limit" (set between $20^{\circ} \mathrm{C}$ and $22{ }^{\circ} \mathrm{C}$ ), the heating system is activated (see Fig. 11(b)) and warms up all rooms at maximum power. If the temperature of a particular room is reaching the "maximum temperature limit" (set between $22^{\circ} \mathrm{C}$ and $24^{\circ} \mathrm{C}$ ), the room's thermostat adjusts the fluid flow in the corresponding UFH loop to keep the temperature at the maximum temperature limit, which decreases accordingly the total fluid flow rate of the MCHP. When the average temperature of the house (defined as the floor area weighted average temperature of all rooms) reaches the maximum temperature limit, the whole heating system is turned off.

The temperature span between the minimum and maximum temperature limits centred on the neutral indoor temperature set point, cannot be greater than $4 \mathrm{~K}$. These requirements maintain a normal thermal comfort level with less than $10 \%$ dissatisfied occupants [65].

One can see in Fig. 11(c) the clear change in the MCHP fluid flow rate profile. When the heating system is activated, the fluid flow rate is kept at its maximum most of the time. Consequently, the COP during operation time is greatly improved (see Fig. 11(d)). 

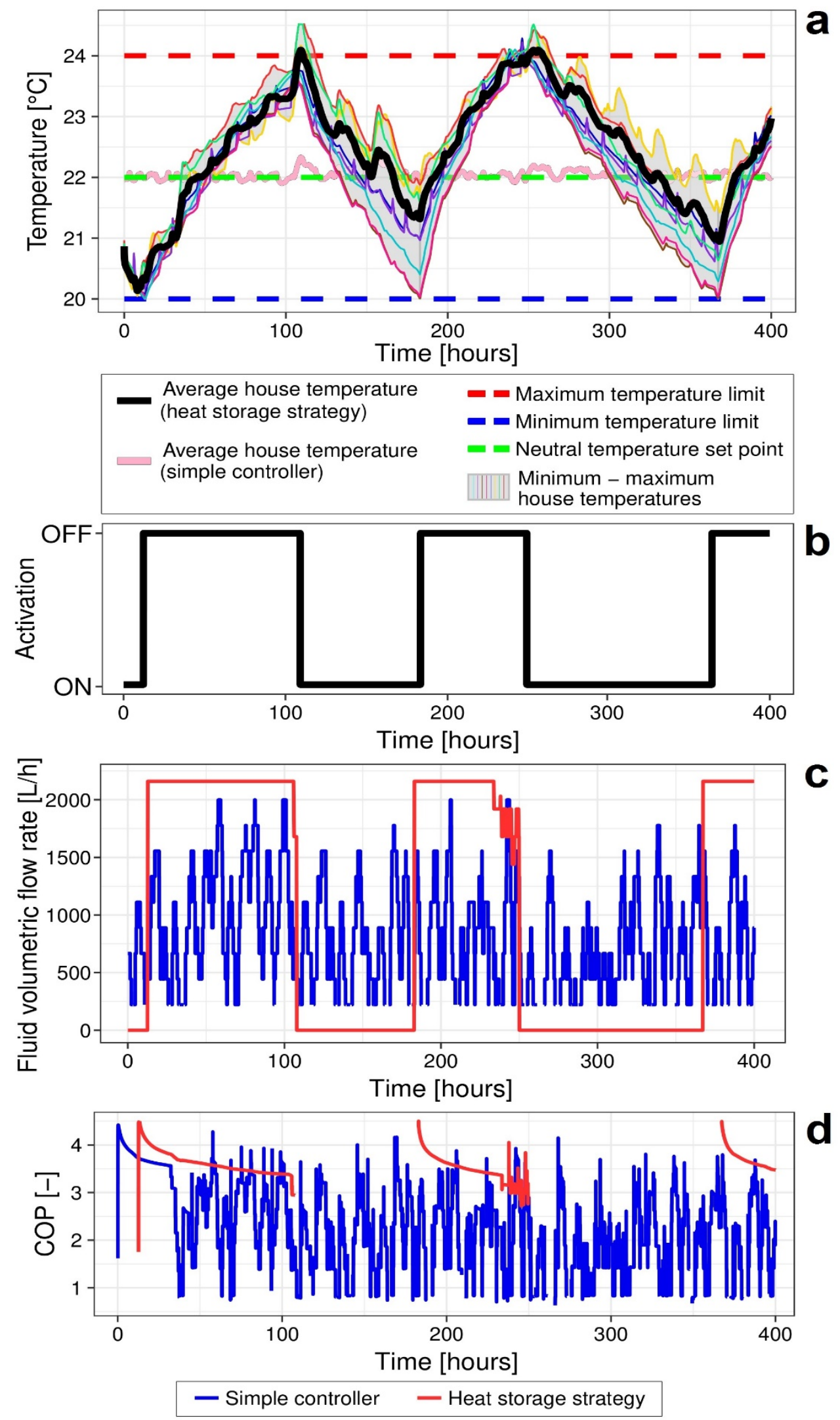

Fig. 11. Example of heat storage strategy control for MCHP: (a) temperatures in the house (with and without heat storage strategy); (b) activation of the MCHP (with heat storage strategy); (c) fluid volumetric flow rate of the MCHP (with and without heat storage strategy); (d) COP of the MCHP (with and without heat storage strategy). 


\section{Results and discussion}

The simulation results presented hereafter correspond to a four-month heating period from the 1st of January to the 30th of April under Danish weather conditions [50]. The "heat storage temperature span" is defined as the difference between the minimum and the maximum temperature limits.

\subsection{Performance metric}

6 The performance of a heat pump system is commonly assessed by calculating its coefficient of performance (COP). In 7 the case of the magnetocaloric device, $C O P_{M C H P}$ is calculated with the useful heating power $Q_{\text {heating }}$ delivered to the 8 UFH, the circulation pump work $W_{\text {pump }}$, the motor work $W_{\text {motor }}$, and the valves work $W_{\text {valves }}$. In the case of the 9 conventional vapour-compression heat pump, $C O P_{C V C H P}$ is calculated with the useful heating power $Q_{\text {heating }}$ delivered to the UFH, the compressor work $W_{\text {compressor }}$, and the work of the 3 circulation pumps $W_{\text {pump }}$.

$$
\begin{gathered}
C O P_{M C H P}=\frac{Q_{\text {heating }}}{W_{\text {pump }}+W_{\text {motor }}+W_{\text {valves }}}(9) \\
C O P_{C V C H P}=\frac{Q_{\text {heating }}}{W_{\text {compressor }}+W_{\text {pump }}}(10)
\end{gathered}
$$

\subsection{Example of magnetocaloric heat pump operation improvement with the heat storage strategy}

In this section, we examine an example of magnetocaloric heat pump operation improvement by using a heat storage strategy. The building case has a medium thermal inertia of $60 \mathrm{Wh} / \mathrm{K} \cdot \mathrm{m}^{2}$, and a heat storage temperature span of $4 \mathrm{~K}$. One can clearly see in Fig. 12 that the heat storage strategy enables a shift of most of the operation time towards maximum fluid flow rate. Consequently, the MCHP is activated less often and for shorter periods of time but with significantly improved COP (see Fig. 13 and Fig. 14). In that case, the average COP of the heating system during the four-month heating period with a simple controller is of $1.81\left(10^{\text {th }}\right.$ and $90^{\text {th }}$ percentile of the COP are 0.83 and 3.20 , respectively). The heat storage strategy improves the average COP to $3.48\left(10^{\text {th }}\right.$ and $90^{\text {th }}$ percentile of the COP are 3.00 and 3.89 , respectively). 


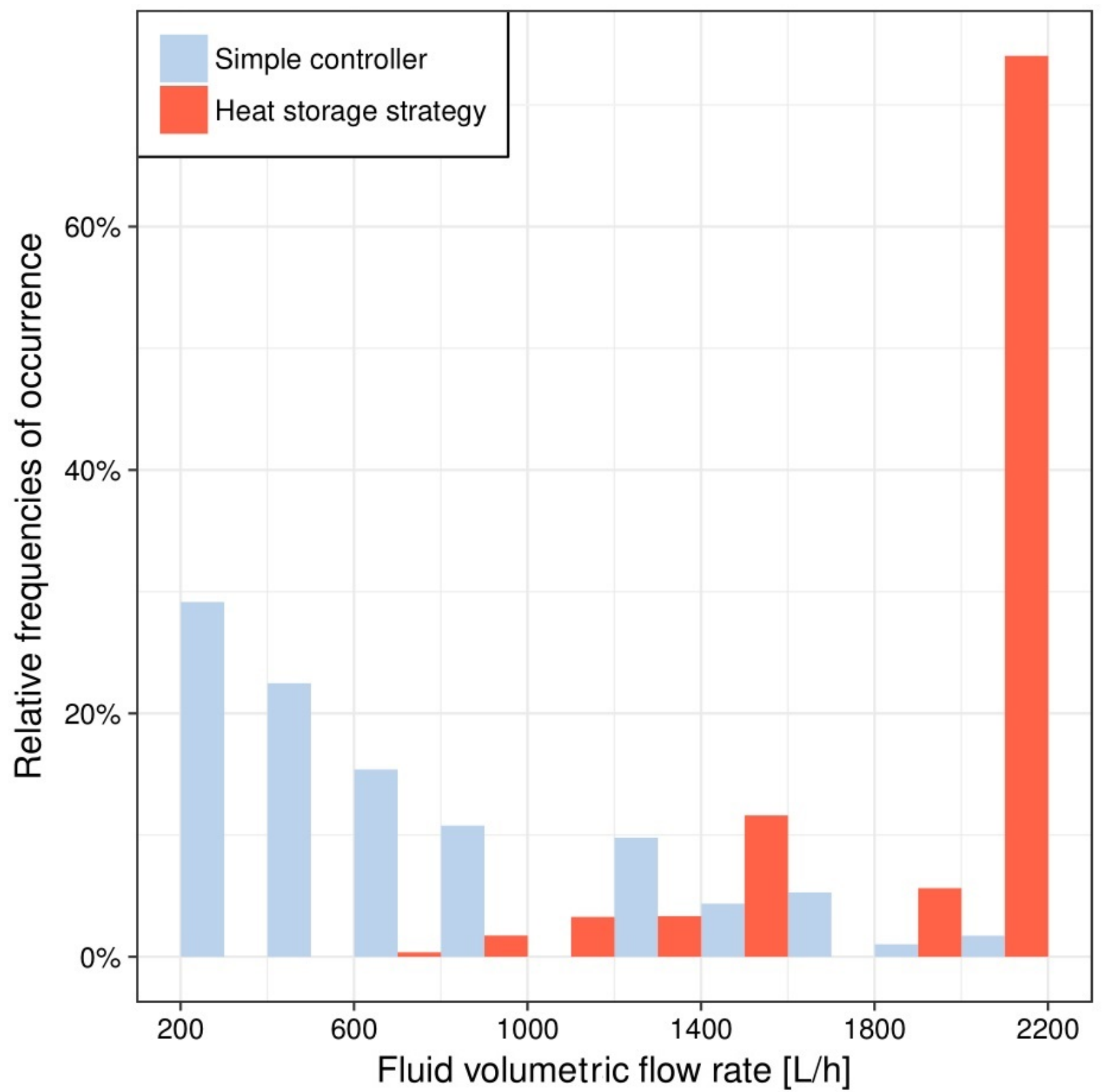

Fig. 12. Histogram of the MCHP heating system fluid volumetric flow rate during the four-month heating test period. 3 


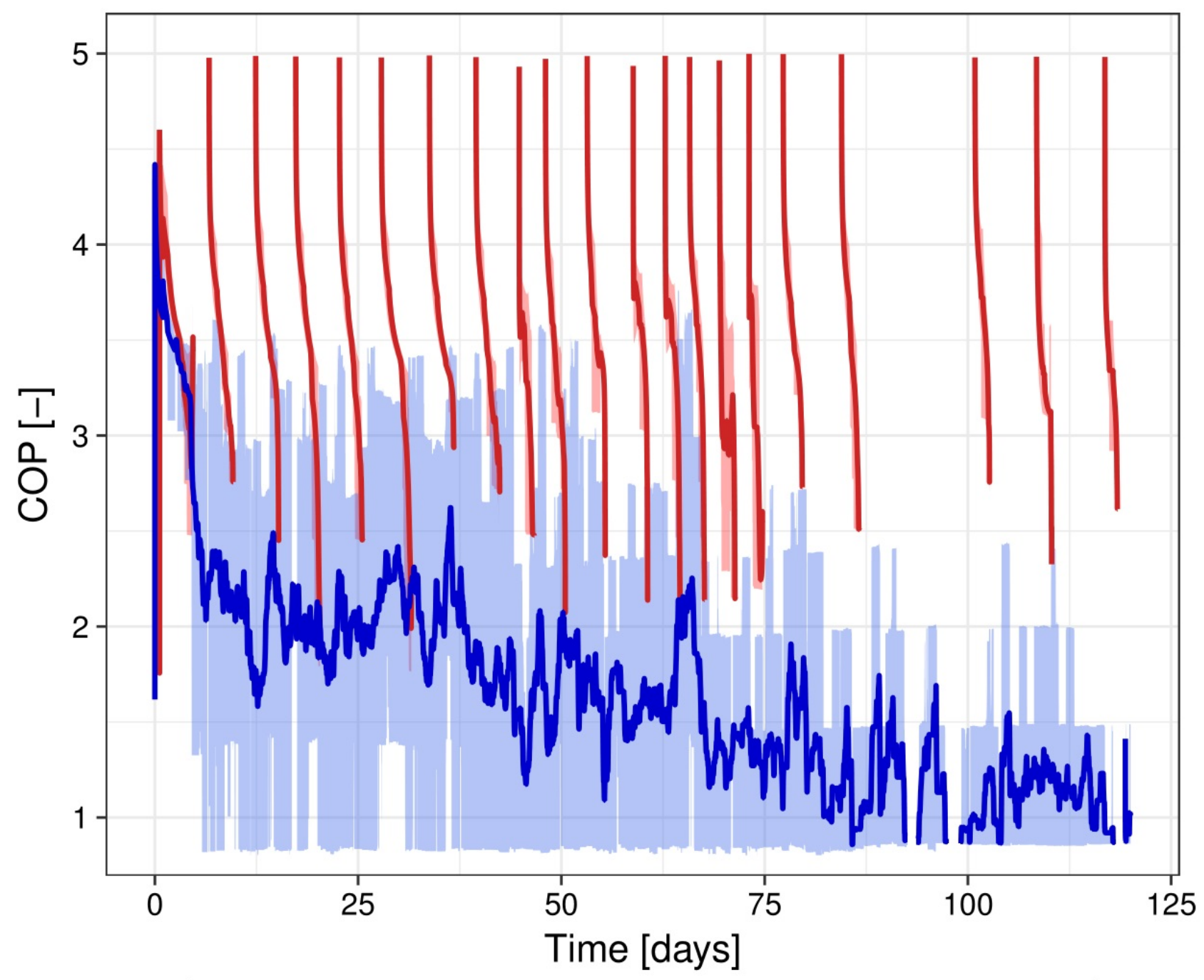

— Daily average COP (simple controller)

$10 \%-90 \%$ percentile COP (simple controller)

- Daily average COP (heat storage strategy)

$10 \%-90 \%$ percentile COP (heat storage strategy)

Fig. 13. COP of the MCHP system as a function of time during the four-month heating test period. 


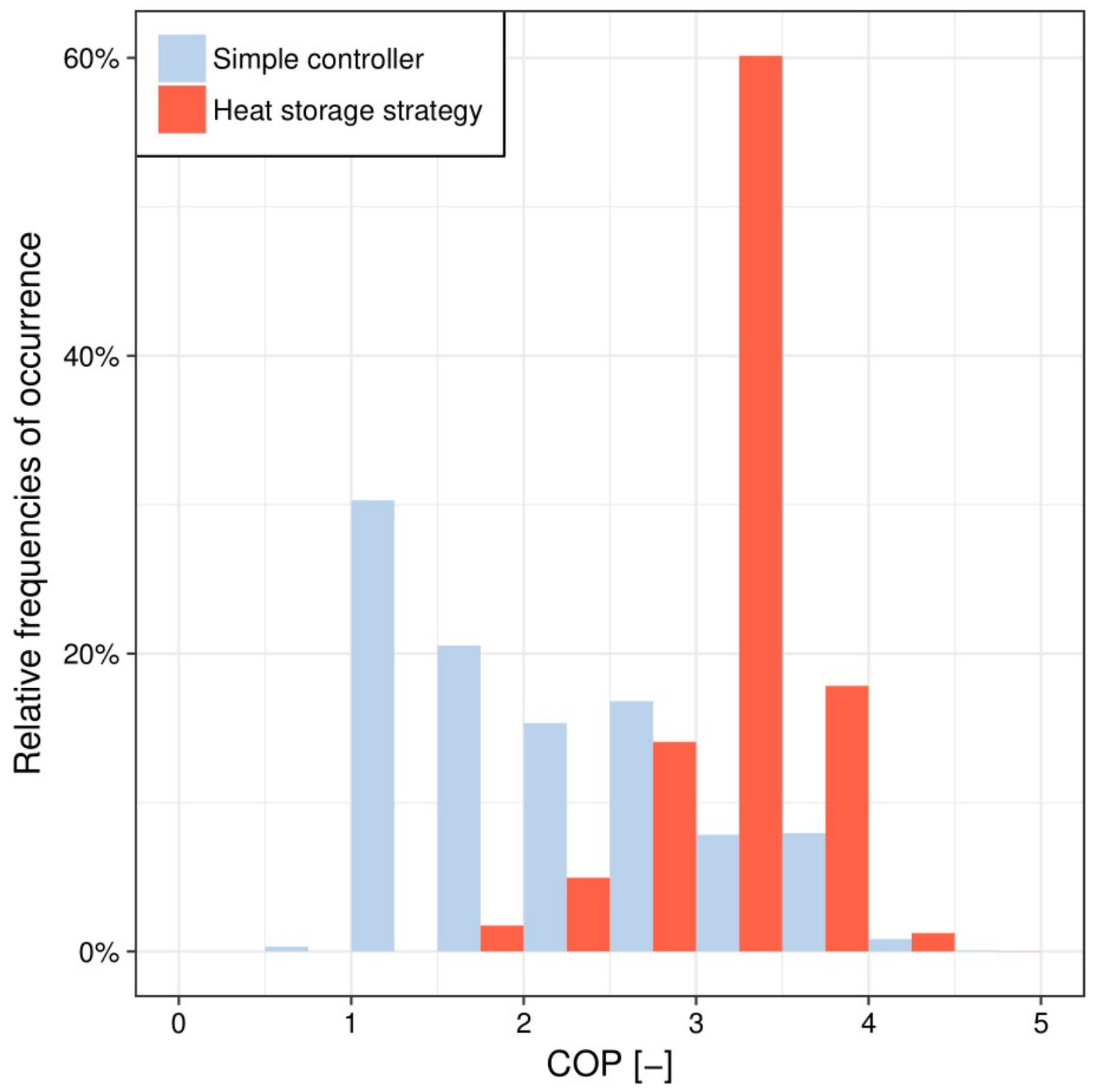

Fig. 14. Histogram of the MCHP heating system COP during the four-month heating test period. 2 

performance

In this section, the results of 18 different study cases are analysed. The MCHP system with a heat storage strategy is compared with the simple controller cases and the conventional VCHP cases. As mentioned before, 3 different classes of building thermal inertia are tested. The heat storage temperature span of the MCHP heat storage strategy is varied from 0 $\mathrm{K}$ to $4 \mathrm{~K}$. The $0 \mathrm{~K}$ temperature span cases actually correspond to the simple controller cases.

One can see in Fig. 15 that both the heat storage temperature span and the thermal inertia have a positive impact on the average fluid flow rate of the heat pump, which improves its performance. Fig. 16 emphasizes the maximization of the time duration at which the heating system runs at full-load and highest COP. In the first part of the MCHP activation period, the heating system runs at full capacity and highest COP. The second part of the MCHP activation period starts when the first room of the house reaches the maximum temperature limit. During that time, the heating system operates at part-load with lower COP until the MCHP is turned off. It is quite clear that the larger the heat storage temperature span and structural thermal inertia, the longer the first part of the activation period at continuous full heating capacity compared to the part-load period.

Fig. 17 presents the consequences of this maximization of the fluid flow rate in terms of COP for the heating system. Larger thermal inertia and heat storage temperature span lead to significant improvements of the COP. One can notice that even a moderate heat storage temperature span of $0.5 \mathrm{~K}$ can increase the average COP by $40 \%, 74 \%$ and $78 \%$ for light, medium and heavy structure houses, respectively. At a maximum heat storage temperature span of $4 \mathrm{~K}$, the average COP reaches $2.90\left(10^{\text {th }}\right.$ and $90^{\text {th }}$ percentile of the COP are 2.11 and 3.44 , respectively) for the light thermal inertia house, $3.48\left(10^{\text {th }}\right.$ and $90^{\text {th }}$ percentile of the COP are 3.00 and 3.89 , respectively) for the medium thermal inertia house, and 3.51 $\left(10^{\text {th }}\right.$ and $90^{\text {th }}$ percentile of the COP are 3.12 and 3.89, respectively) for the heavy thermal inertia house. In the cases of medium and heavy houses, the MCHP with heat storage strategy presents performances which are comparable to those of the conventional VCHP system. However, the COP improvement of the MCHP is very limited for a heat storage temperature span above $2 \mathrm{~K}$. In addition, it can be noted that medium and heavy thermal mass houses have very similar performances. The latter can be explained by the fact that the light-weight house cases have their UFH circuits embedded in a light-weight structure wooden floor, whereas it is a concrete screed UFH for medium and heavy-weight houses. The UFH loops enable an important thermal activation of the floor elements. Consequently, a concrete floor provides a much larger thermal storage potential than a wooden one. However, additional structural thermal mass such as concrete or brick walls and ceilings are not directly activated by the UFH. Therefore, its benefit in terms of MCHP operation improvement is limited.

Because no building envelope insulation is perfect, accumulating thermal energy in the indoor environment by increasing the building's internal temperature will necessarily lead to higher heat losses by transmission and ventilation. However, one can see in Fig. 18 that the improvement of the heating system COP over-compensates the envelope additional heat losses. This leads to a substantial decrease of the total energy use during the heating period of about $28 \%$ to $41 \%$. In the cases of medium and heavy-weight houses, the total energy usage of the MCHP system with heat storage strategy is thereby similar to the conventional VCHP.

Another constraint of TES in the indoor space by means of temperature set point modulation is the variability of the operative temperature. To avoid thermal discomfort, the indoor temperature should not change faster than $2.1 \mathrm{~K} / \mathrm{h}$ [66]. This requirement is fulfilled in every case here. However, the increase of temperature set point to $24^{\circ} \mathrm{C}$ in well-insulated south-oriented rooms with large windows could increase the risks of discomfort due to over-heating during sunny days. Fig. 19 illustrates this indoor temperature variability with the calculation of the difference between the $5^{\text {th }}$ and $95^{\text {th }}$ percentile of all rooms operative temperature during the four-month heating test period. Naturally, the temperature variability increases with the heat storage temperature span and decreases with building thermal inertia. It can be noted for medium and heavy-weight structure houses, the temperature variability with heat storage strategy never exceeds the one of the simple controller reference case, even with the maximum heat storage temperature span of $4 \mathrm{~K}$. 


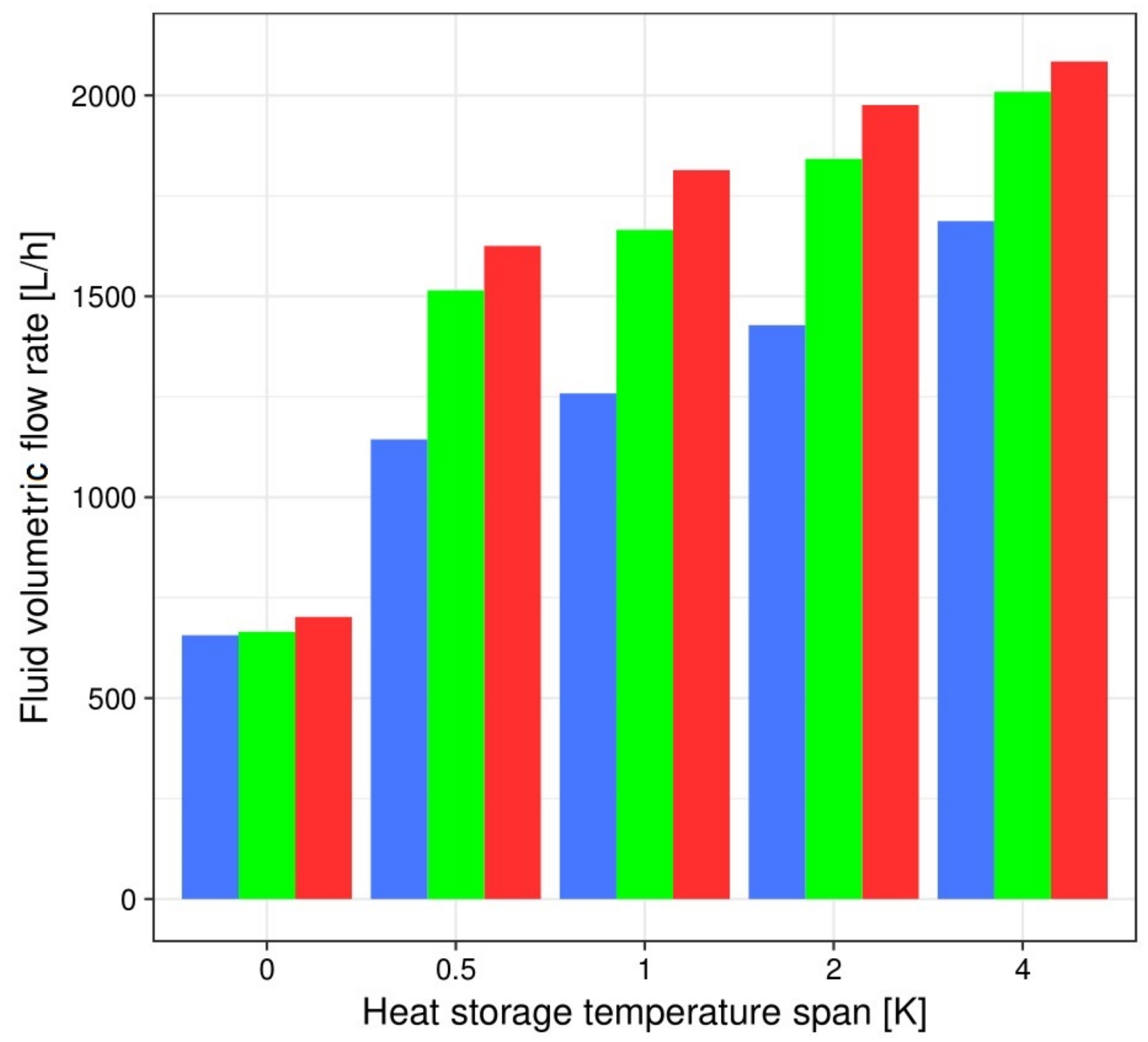

Building thermal inertia: Light $\square$ Medium $\square$ Heavy

Fig. 15. Average fluid volumetric flow rates of the MCHP during the four-month heating test period. 


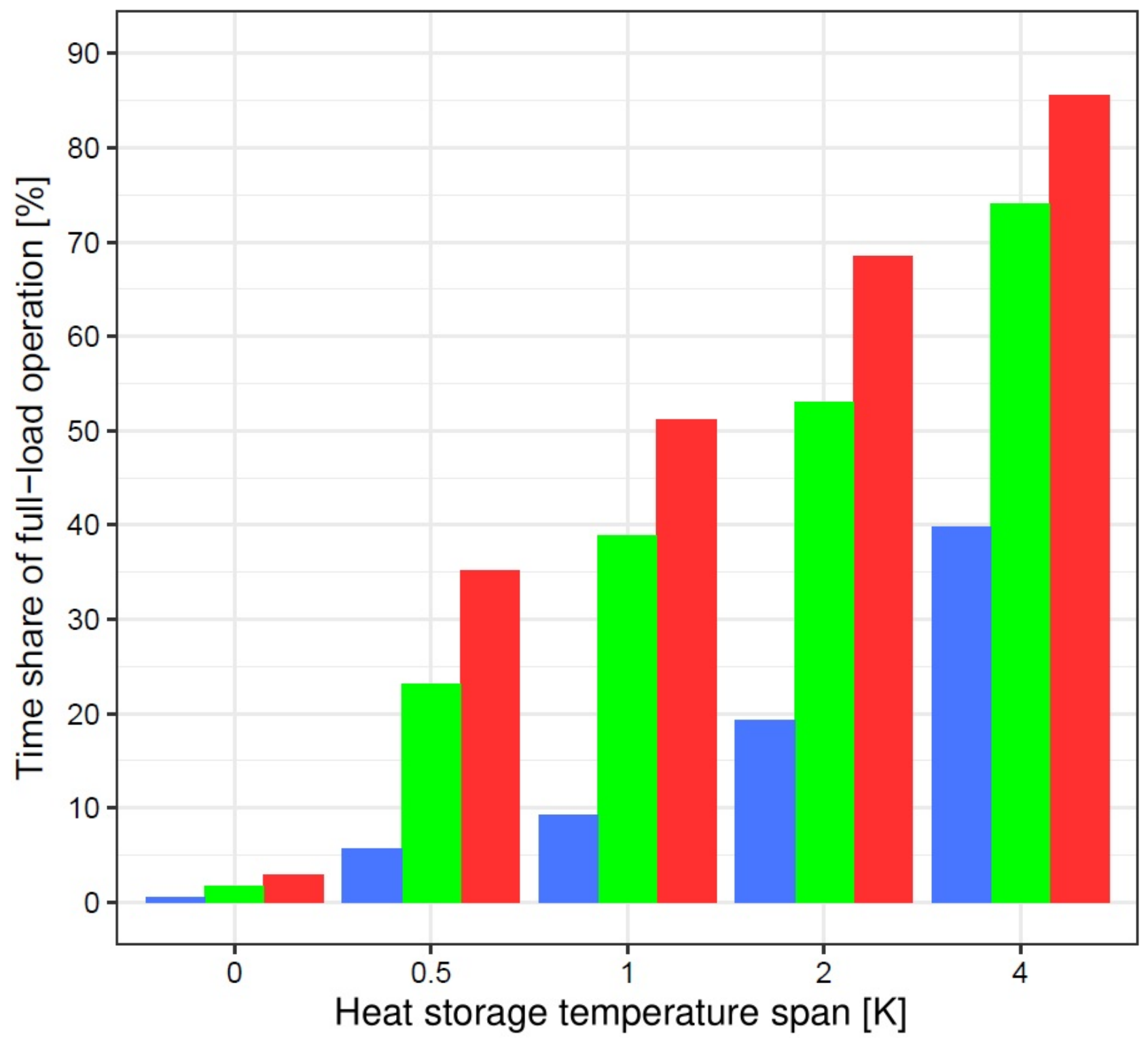

\section{Building thermal inertia: Light $\square$ Medium $\square$ Heavy}

Fig. 16. Full-load operation time share of the MCHP during the four-month heating test period. 

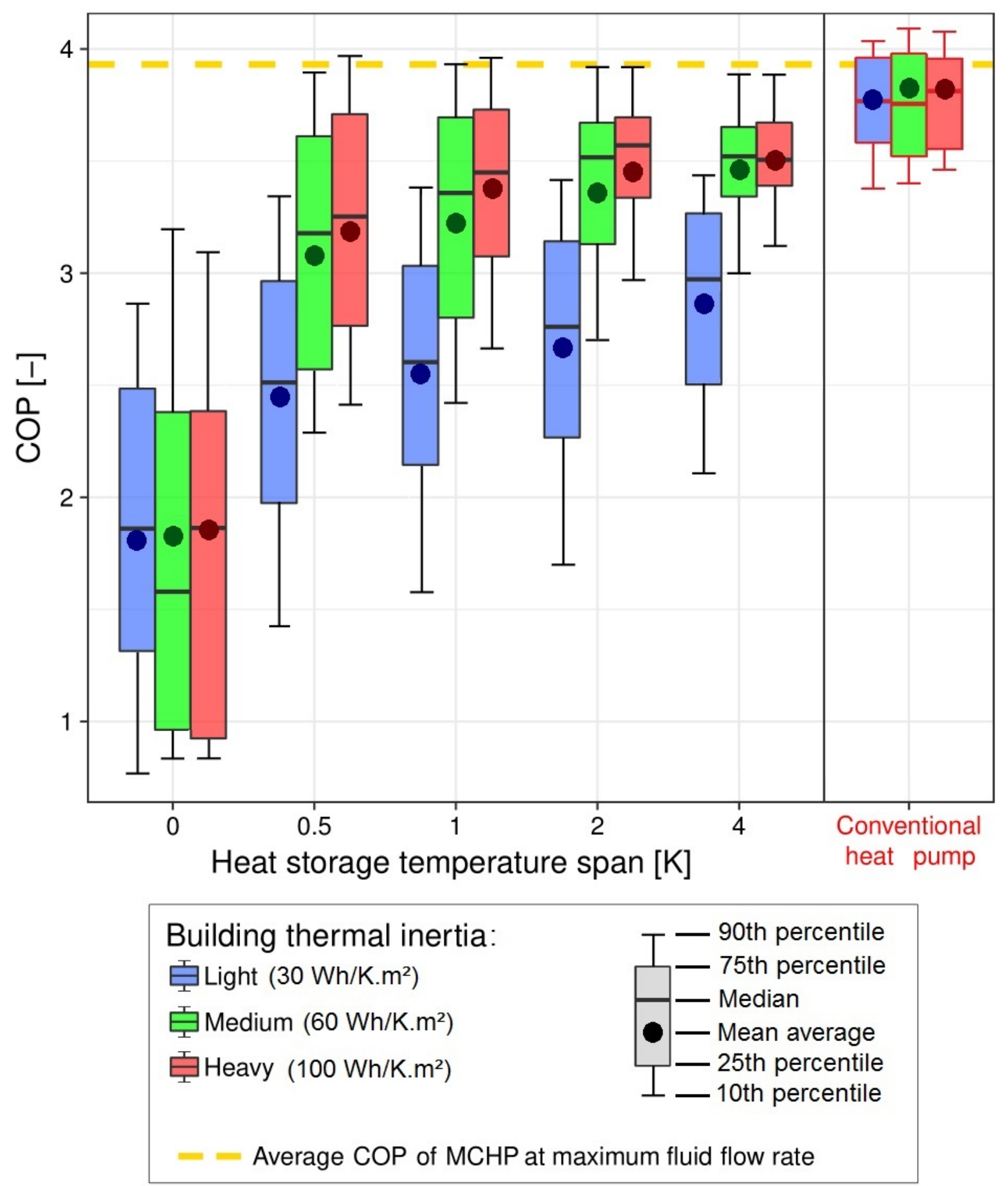

Fig. 17. Box plot diagram of the heat pump COP as a function of heat storage temperature span and building thermal inertia (MCHP on the left, conventional vapour-compression heat pump on the right). 


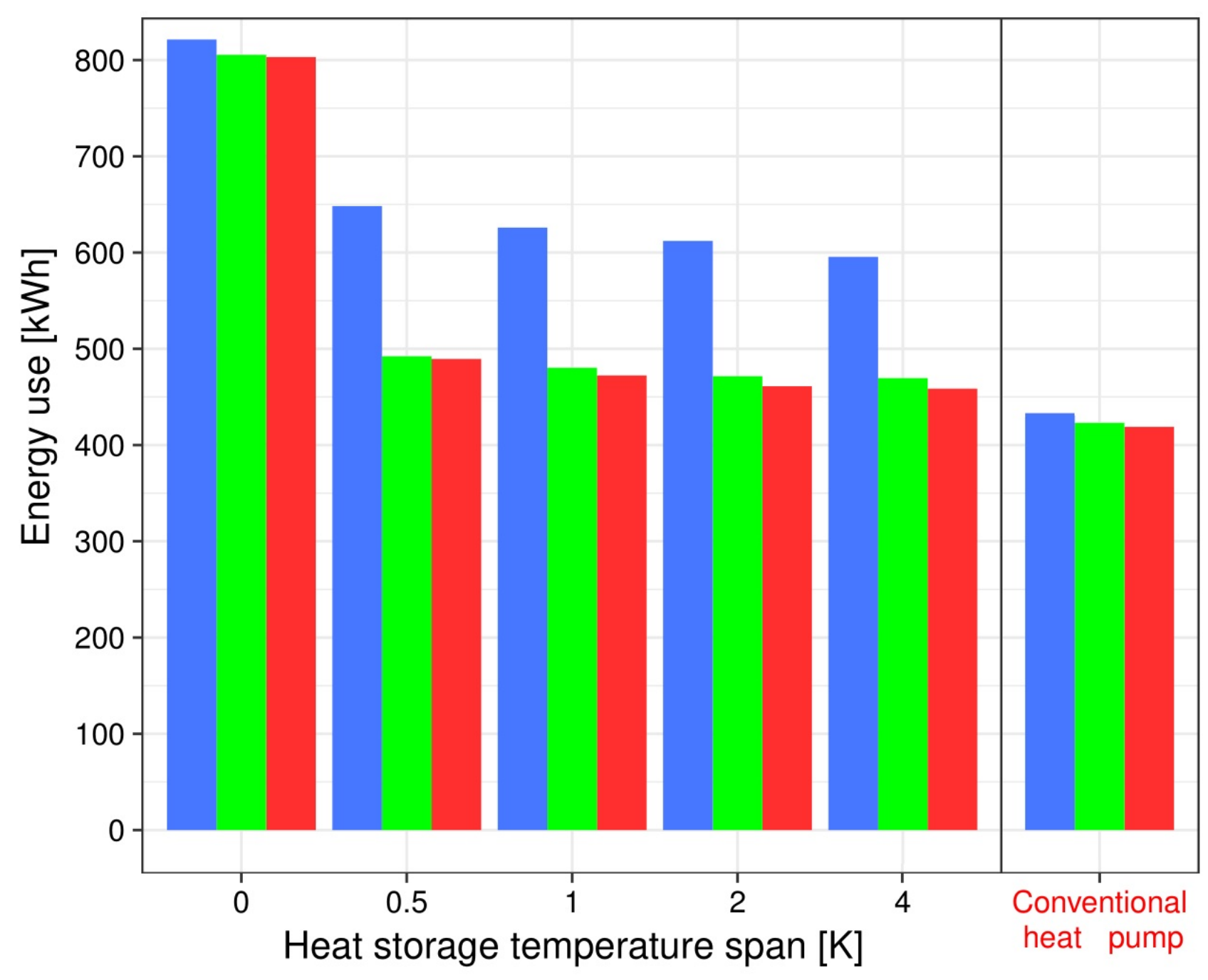

\section{Building thermal inertia: $\square$ Light $\square$ Medium $\square$ Heavy}

Fig. 18. Total heating use during the four-month heating test period (MCHP on the left, conventional vapourcompression heat pump on the right). 


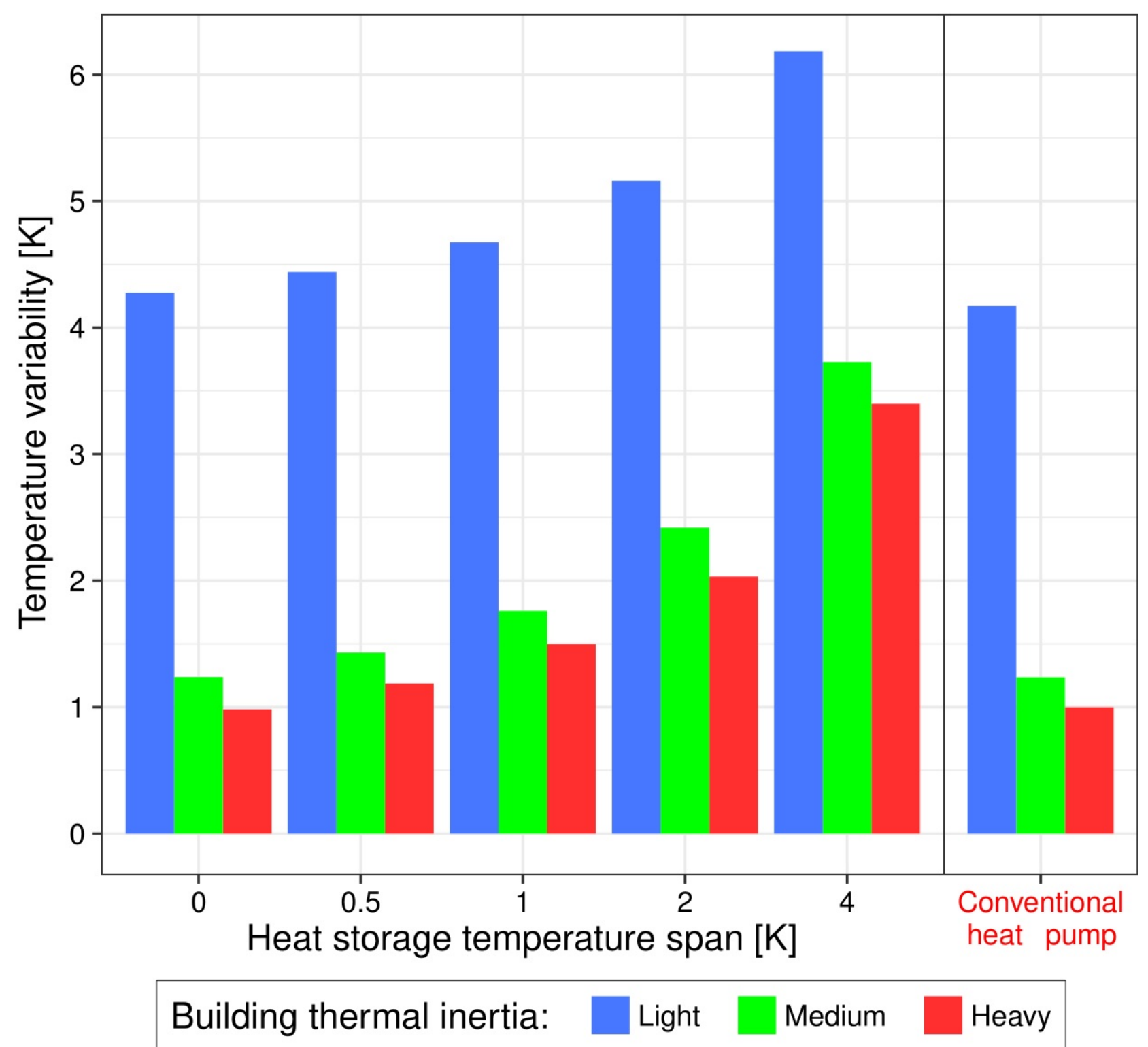

Fig. 19. Temperature variability (difference between $5^{\text {th }}$ and $95^{\text {th }}$ percentile of the house temperature) during the fourmonth heating test period (MCHP on the left, conventional vapour-compression heat pump on the right). 
This numerical study has tested the use of a heat storage strategy in order to improve the operation performance of a magnetocaloric heat pump integrated in a low-energy Danish residential building. This innovative heating system can be implemented in a single hydronic loop including a vertical borehole ground source heat exchanger and a radiant underfloor heating without intermediate heat exchanger or hot water storage tank. Because the magnetocaloric heating system presents a maximum coefficient of performance at system's maximum fluid flow rate (COP of 3.93), it is beneficial to minimize its part-load operation time. In conventional heat pump configurations, the hot water storage tank allows a certain power generation flexibility which reduces the number of ON/OFF cycles and improves global performances. In the case of the magnetocaloric system, the building thermal inertia is employed to enable this energy flexibility. Indoor temperature set point modulation is used to store heat in the built environment and building thermal mass, and thus maximize the full-load operation time. Heating needs are shifted in time and concentrated over longer periods where the magnetocaloric device can run at full capacity with highest COP.

Both the building thermal inertia and the heat storage temperature span (difference between upper and lower limit for the temperature set point modulation controller) have positive impacts on the magnetocaloric system performance. A moderate heat storage temperature span of $0.5 \mathrm{~K}$ can increase the average COP by $40 \%, 74 \%$ and $78 \%$ for light, medium and heavy structure houses, respectively. With a maximum temperature set point modulation of $4 \mathrm{~K}$, the average COP reaches $2.90,3.48$ and 3.51 for light, medium and heavy structure houses, respectively. These performances are comparable with conventional vapour-compression heat pump installations. Despite the increase of transmission and ventilation losses, the heat storage strategy decreased the total energy use by about $28 \%$ to $41 \%$ during the four-month heating test period.

Conclusions can be drawn about the impact of the building thermal mass and heat storage temperature span on the magnetocaloric heat pump performance. The thermal mass of the concrete screed where the under-floor heating circuit is laid, brings great benefits compared to a light-weight wooden floor. This large thermal inertia is well thermally activated and offers good heat storage for higher average heat pump COP. However, additional thermal mass of walls and ceilings has only limited impact because these construction elements are not directly activated by the under-floor heating system. Concerning the temperature set point modulation controller, a temperature span above $2 \mathrm{~K}$ does not improve the heat pump operation much, whereas it significantly increases the temperature variability in the dwelling.

This article has demonstrated the possibility of using heat storage in the indoor environment as an effective strategy to improve the operation of the magnetocaloric heat pump. It can significantly improve the average COP of the entire heating system and reduce the energy usage, but, as a trade-off, decreases the building temperature stability, augmenting the risks of over-heating and thermal discomfort.

Further research should be carried out on new control strategies and building integration possibilities for this innovative magnetocaloric device. The heat storage in the indoor environment is built upon the relatively large range of human thermal comfort. However, the acceptability of the occupants regarding automated temperature set point modulation should be tested, especially for dwellings. The robustness of such a control strategy should also be further studied in the case of buildings with critical rooms which cool down much faster than the others. Finally, cascading configurations for the magnetocaloric heat pump could generate much larger temperature span between heat source and heat sink, allowing its use domestic hot water production.

\section{Acknowledgements}

This work was financed by the ENOVHEAT project which is funded by Innovation Fund Denmark (contract no 12- 
[1] United Nations Framework Convention on Climate Change, Report of the Conference of the Parties on its twenty-first session, held in Paris from 30 November to 13 December 2015 - Adoption of the Paris Agreement, 2015.

[2] European Climate Foundation, Roadmap 2050: a practical guide to a prosperous, low-carbon Europe, Technical report, 2010.

[3] Building Performance Institute Europe (BPIE), Europe's Buildings under the Microscope, Executive Summary 2011. http://bpie.eu/wpcontent/uploads/2015/10/HR_EU_B_under_microscope_study.pdf, 2011 (accessed 24 January 2018).

[4] S.J. Self, B.V. Reddy, M.A. Rosen, Geothermal heat pump systems: Status review and comparison with other heating options, Applied Energy 111 (2013) 341-348.

[5] D. Fischer, H. Madani, On heat pumps in smart grids: A review, Renewable and Sustainable Energy Reviews 70 (2017) 342-357.

[6] H. Lund, B. Möller, B.V. Mathiesen, A. Dyrelund, The role of district heating in future renewable energy systems, Energy 35 (3) (2010) $1381-90$.

[7] A. Palzer, H.M. Henning, A comprehensive model for the German electricity and heat sector in a future energy system with a dominant contribution from renewable energy technologies - Part II: Results, Renewable and Sustainable Energy Reviews 30 (2014) 1019-1034.

[8] J. Cockroft, N. Kelly, A comparative assessment of future heat and power sources for the UK domestic sector, Energy Conversion and Management 47 (2006) 2349-2360.

[9] T. Nowak, P. Westring, The European Heat Pump Association AISBL (EHPA), European heat pump market and statistics report 2015. http://www.ehpa.org/fileadmin/red/07._Market_Data/2014/EHPA_European_Heat_Pump_Market_and_Statistics_Report_2015__executive_Summary.pdf, 2015 (accessed 24 January 2018).

[10] W. Goetzler, R. Zogg, J. Young, C. Johnson, Energy savings potential and RD\&D opportunities for non-vapor compression HVAC technologies, U.S. Department of Energy, Building Technologies Office. http://energy.gov/sites/prod/files/2014/03/f12/NonVapor\%20Compression\%20HVAC\%20Report.pdf, 2014 (accessed 10 September 2018).

[11] B. Neese, B. Chu, S.G. Lu, Y. Wang, E. Furman, Q.M. Zhang, Large electrocaloric effect in ferroelectric polymers near room temperature, Science 321 (2008) 821-823.

[12] U. Plaznik, M. Vrabelj, Z. Kutnjak, B. Malič, B. Rožič, A. Poredoš, A. Kitanovski, Numerical modelling and experimental validation of a regenerative electrocaloric cooler, International Journal of Refrigeration 98 (2019) 139-149.

[13] L. Mañosa, D. González-Alonso, A. Planes, E. Bonnot, M. Barrio, J.L. Tamarit, S. Aksoy, M. Acet, Giant solid-state barocaloric effect in the NiMn-In magnetic shape-memory alloy, Nature Materials 9 (2010) 478-481.

[14] E. Bonnot, R. Romero, L. Mañosa, E. Vives, A. Planes, Elastocaloric Effect Associated with the Martensitic Transition in Shape-Memory Alloys, Physical Review Letters 100 (2008) 125901.

[15] K. Engelbrecht, D. Eriksen, S. Dall'Olio, J. Tušek, N. Pryds, A regenerative elastocaloric heat pump, Nature Energy 1 (2016) 16134.

[16] A. Smith, C.R.H. Bahl, R. Bjørk, K. Engelbrecht, K.K. Nielsen, N. Pryds, Materials challenges for high performance magnetocaloric refrigeration devices, Advanced Energy Materials 2 (2012) 1288-1318.

[17] A. Kitanovski, J. Tušek, U. Tomc, U. Plaznik, M. Ožbolt, A. Poredoš, Magnetocaloric Energy Conversion: From Theory to Applications, Springer International Publisher, New York, 2015.

[18] C. Zimm, A. Boeder, B. Mueller, K. Rule, S.L. Russek, The evolution of magnetocaloric heat-pump devices, MRS Bulletin 43 (2018) $274-279$.

[19] P. Weiss, A. Piccard, Sur un nouveau phénomène magnétocalorique, Comptes Rendus Acad. Sci. (Paris) 166 (1918) 352-354.

[20] P. Weiss, R. Forrer, Aimantation et phénomène magnetocalorique du nickel, Annales de Physique (Paris) 5 (1926) 153-213.

[21] P. Debye, Einige Bemerkungen zur Magnetisierung bei tiefer Temperatur, Annalen der Physik (Leipzig) 386 (1926) 1154-1160.

[22] W.F. Giauque, A thermodynamic treatment of certain magnetic effects. A proposed method of producing temperatures considerably below 1 absolute, Journal of the American Chemical Society 49 (1927) 1864-1870.

[23] W.F. Giauque, D.P. MacDougall, The production of temperatures below one degree absolute by adiabatic demagnetization of gadolinium sulfate, Journal of the American Chemical Society 57 (1935) 1175-1185.

[24] G.V. Brown, Magnetic heat pumping near room temperature, Journal of Applied Physics 47 (1976) 3673.

[25] J.A. Barclay, W.A. Steyert, US Patent US4332135 (1985).

[26] F.C. Chen, R.W. Murphy, V.C. Mei, G.L. Chen, Thermodynamic Analysis of Four Magnetic Heat-Pump Cycles, Journal of Engineering for Gas Turbines and Power 114 (1992) 715720

[27] C. Zimm, A. Jastrab, V. Sternberg, V. Pecharsky, K. Gschneidner Jr, M. Osborne, I. Anderson, Description and performance of a near room temperature magnetic refrigerator, Advances in Cryogenic Engineering 43 (1998) 1759-1766.

[28] K. Engelbrecht, D. Eriksen, C.R.H. Bahl, R. Bjørk, J. Geyti, J.A. Lozano, K.K. Nielsen, F. Saxild, A. Smith, N. Pryds, Experimental results for a novel rotary active magnetic regenerator, International Journal of Refrigeration 35 (2012) 1498-1505.

[29] T. Okamura, N. Hirano, Improvement of the performance of a room temperature magnetic refrigerator using Gd-alloy, Journal of the Japan Society of Applied Electromagnetics and Mechanics 21 (2013) 10-14.

[30] S. Jacobs, J. Auringer, A. Boeder, J. Chell, L. Komorowski, J. Leonard, S. Russek, C. Zimm, The performance of a large-scale rotary magnetic refrigerator, International Journal of Refrigeration 37 (2014) 84-91.

[31] C. Aprea, A. Greco, A. Maiorino, GeoThermag: A geothermal magnetic refrigerator, International Journal of Refrigeration 59 (2015) 75-83.

[32] C. Aprea, A. Greco, A. Maiorino, C. Masselli, The energy performances of a rotary permanent magnet magnetic refrigerator, International Journal of Refrigeration 61 (2015) 1-11.

[33] J.A. Lozano, M.S. Capovilla, P.V. Trevizoli, K. Engelbrecht, C.R.H. Bahl, J.R. Barbosa Jr, Development of a novel rotary magnetic regenerator, International Journal of Refrigeration 68 (2016) 187-197.

[34] D. Eriksen, K. Engelbrecht, C.R.H. Bahl, R. Bjørk, Exploring the efficiency potential for an active magnetic regenerator, Science and Technology for the Built Environment 22 (2016) 527533

[35] T. Lei, K. Engelbrecht, K.K. Nielsen, C.T. Veje, Study of the geometries of active magnetic regenerators for room temperature magnetocaloric refrigeration, Applied Thermal Engineering 111 (2017) 1232-1243.

[36] C.R.H. Bahl, EnovHeat project summary: development of efficient novel magnetocaloric heat pumps. http://www.enovheat.dk/Research/ProjectSummary, 2015 (accessed 24 January 2018).

[37] H. Johra, K. Filonenko, P. Heiselberg, C. Veje, T. Lei, S. Dall'Olio, K. Engelbrecht, C. Bahl, Integration of a magnetocaloric heat pump in a lowenergy residential building, Building Simulation (2018). 
[38] K. Engelbrecht, C.R.H. Bahl, Evaluating the effect of magnetocaloric properties on magnetic refrigeration performance, Journal of Applied Physics 108 (2010) 123918.

[39] S. Dall'Olio, T. Lei, K. Engelbrecht, C.R.H. Bahl, The effect of tapering on a magnetocaloric regenerator bed, International Journal of Refrigeration 84 (2017) 300-308.

[40] P. Li, M. Gong, G. Yao, J. Wu, A practical model for analysis of active magnetic regenerative refrigerators for room temperature applications, International Journal of Refrigeration 29 (2006) 1259-1266.

[41] S. Qian, L. Yuan, J. Yu, G. Yan, Variable load control strategy for room-temperature magnetocaloric cooling applications, Energy 153 (2018) 763-755.

[42] European Commission, European SmartGrids Technology Platform, Vision and Strategy for Europe's Electricity Networks of the Future. https://ec.europa.eu/research/energy/pdf/smartgrids en.pdf, 2006 (accessed 20 October 2018).

[43] B.V. Mathiesen, H. Lund, D. Connolly, H. Wenzel, P.A. Østergaard, B. Möller, S. Nielsen, I. Ridjan, P. Karnøe, K. Sperling, F.K. Hvelplund, Smart Energy Systems for coherent 100\% renewable energy and transport solutions, Applied Energy 145 (2015) 139-154.

[44] S.J. Østergaard, A. Marszal-Pomianowska, R. Lollini, W. Pasut, A. Knotzer, P. Engelmann, A. Stafford, G. Reynders, IEA EBC Annex 67 Energy Flexible Buildings, Energy and Buildings 155 (2017) 25-34.

[45] K. Hedegaard, B.V. Mathiesen, H. Lund, P. Heiselberg, Wind power integration using individual heat pumps - Analysis of different heat storage options, Energy 47 (2012) 284-93.

[46] J. Le Dréau, P. Heiselberg, Energy flexibility of residential buildings using short term heat storage in the thermal mass, Energy 111 (2016) 991 1002.

[47] T. Lei, K. Navickaitè, K. Engelbrecht, A. Barcza, H. Vieyra, K.K Nielsen, C.R.H. Bahl, Passive characterization and active testing of epoxy bonded regenerators for room temperature magnetic refrigeration, Applied Thermal Engineering 128 (2018) 10-19.

[48] The Danish Ministry of Economic, Business Affairs Enterprise, and Construction Authority, Building Regulations. http://bygningsreglementet.dk/file/155699/BR10_ENGLISH.pdf, 2010 (accessed 24 January 2018).

[49] H. Johra, P. Heiselberg, J. Le Dréau, Influence of envelope, structural thermal mass and indoor content on the building heating energy flexibility, Energy and Buildings 183 (2019) 325-339.

[50] P.G. Wang, M. Scharling, K.P. Nielsen, K.B. Wittchen, C. Kern-Hansen, Danish Ministry of Climate, Energy and Building, $2001-2010$ Danish Design Reference Year - Reference climate dataset for technical dimensioning in building, construction and other sectors, Technical Report 13$19,2013$.

[51] R.L. Jensen, J. Nørgaard, O. Daniels, R.O. Justesen, Person-og forbrugsprofiler: bygningsintegreret energiforsyning, DCE Technical Reports; Nr. 69. http://vbn.aau.dk/files/63284701/Person_og_Forbrugsprofiler.pdf, 2011 (accessed 24 January 2018).

[52] H. Johra, P. Heiselberg, Description and Validation of a MATLAB-Simulink Single Family House Energy Model with Furniture and Phase Change Materials, $\quad$ DCE $\quad$ Technical $\quad$ Reports; $\quad$ No. 187. http://vbn.aau.dk/files/244109005/Description_and_Validation_of_a_MATLAB_Simulink_Single_Family_House_Energy_Model_with_Furnit ure and Phase Change Materials.pdf, 2016 (accessed 24 January 2018).

[53] European Committee for Standardization, EN 1264:2011 - Water based surface embedded heating and cooling systems, 2011.

[54] International Organization for Standardization, ISO 11855:2012 - Building environment design. Design, dimensioning, installation and control of embedded radiant heating and cooling systems, 2012.

[55] Uponor GmbH, Heating and cooling solutions - Technical guidelines, 2008.

[56] Verlag des Vereins Deutscher Ingenieure, VDI 4640:2001, Thermal use of the underground-Ground source heat pump systems, 2001.

[57] Uponor GmbH, Ground Energy Technical Information, 2012.

[58] RETScreen International, Minister of Natural Resources Canada, Ground-Source Heat Pump Project Analysis. Canada, 2005.

[59] ClimateMaster, Tranquility Water-to-Water (TMW) Series Submittal Data Model TMW036 - $340 \quad 50 \mathrm{~Hz}-\mathrm{HFC}-410 \mathrm{~A}$. http://lenergy.hu/dokuk/termekek/103/98caovp2.pdf, 2012 (accessed 24 January 2018).

[60] M. Scarpa, K. Grau, B.W. Olesen, Development and validation of a versatile method for the calculation of heat transfer in water-based radiant systems, in: Proceedings of the 11th International Building Performance Simulation Association Conference and Exhibition (BS2009), Glasgow, Scotland, 2009

[61] University of Wisconsin-Madison Solar Energy Laboratory, TRANSSOLAR Energietechnik GmbH, CSTB, TESS, Type 31: Pipe Or Duct, in: TRNSYS 17 - Mathematical Reference, 2012, pp. 186-188

[62] H.J.G. Diersch, D. Bauer, W. Heidemann, W. Rühaak, P. Schätzl, Finite element modeling of borehole heat exchanger systems - Part 1. Fundamentals, Computers and Geosciences 37 (2011) 1122-1135.

[63] G. Angrisani, M. Canelli, C. Roselli, M. Sasso, Calibration and validation of a thermal energy storage model: influence on simulation results, Applied Thermal Engineering 67 (2014) 190-200.

[64] ANSI/ASHRAE Standard, Standard 140-2011: Standard method of test for the evaluation of building energy analysis computer programs, 2011.

[65] European Committee for Standardization, EN ISO 7730 - Ergonomics of the thermal environment-analytical determination of thermal comfort by using calculation of the PMV and PPD indices and local thermal comfort criteria, 2005.

[66] ASHRAE Standard, Standard 55 - 2004: Thermal environmental conditions for human occupancy, 2004. 